\title{
Spectral Band Structure of Periodic Schrödinger Operators on a Generalized Degenerate Zigzag Nanotube
}

\author{
Hiroaki NIIKUNI \\ Maebashi Institute of Technology \\ (Communicated by K. Yoshitomi)
}

\begin{abstract}
We refer generalized degenerate zigzag nanotubes as periodic metric graphs which consist of segments of length 1 and rings of length 2 throughout this paper. In this paper, we consider the case where there are one segment and three rings in the basic period cell and analyze the spectrum of periodic Schrödinger operators on the generalized degenerate zigzag nanotube. We obtain the relationship between the structure of the metric graph and the nondegenerate spectral gaps of the Schrödinger operators.
\end{abstract}

\section{Introduction}

In this paper, we discuss a spectral problem for a quantum graph originating from carbon nanotubes. First, quantum graph is defined in [1] as a triplet of a metric graph, a differential operator and an appropriate vertex condition (for example, Kirchhoff vertex condition, $\delta$ vertex condition and so on. See also [4, 12].). In this paper, we define Schrödinger operators with periodic potentials and Kirchhoff vertex conditions on a periodic quasi-1-dimensional metric graph consisting of lines of length 1 and rings of length 2 and investigate their spectra. We call the metric graph the generalized degenerate zigzag nanotube throughout this paper. We give more precise definition in the next paragraph. Although we later describe the relationship between this paper and $[11,16]$ in detail, we now introduce earlier paper $[2,3,5,11,13,18]$ on the subject of periodic Schrödinger operators on metric graphs in brief before we define our operators. One example of quasi-1-dimensional metric graph is a homogeneous tree, whose vertices have a common number of edges. Carlson [3] analyzed the spectra of periodic Schrödinger operators with $\delta$ vertex conditions on the homogeneous trees by using one-dimensional tools for Hill operators like the monodromy matrix and the Lyapunov function. Since a homogeneous tree does not have any loop, our graph possessing rings is not included in this class. Spectral analysis of periodic Schrödinger operators on metric graphs consisting of loops is seen in [2]. Duclos, Exner and Turek gave spectral

Received May 12, 2014; revised June 3, 2015

Mathematics Subject Classification: 34L05, 34L15, 34B45

Key words and phrases: quantum graph, Schrödinger operator, metric graph, spectral gap, band structure, Hill operator, coexistence problem, carbon nanotube, zigzag nanotube 
results on the straight case and the bending case of the metric graph with $\delta$ vertex conditions. As one of attractive graphs, there are the hexagonal lattices. Physically, the hexagonal lattice corresponds to the graphene, which is an allotrope of carbon. As for the carbon nanotubes, which are also allotropes of carbon with cylindrical structure, Korotyaev and Lobanov [11] and Kuchment and Post [13] established the spectral theory. Especially, it is effective for spectral analysis of periodic Schrödinger operators with Kirchhoff vertex conditions on zigzag nanotubes to consider the so-called degenerate zigzag nanotube, which is the periodic metric graph possessing one line of length 1 and one ring of length 2 in the basic period cell (see also [1] for the carbon nanotube and [9] for the magnetic case). In this paper, we generalize the degenerate zigzag nanotube and investigate the spectra of Schrödinger operators on it. The spectrum of the Schrödinger operator on the zigzag nanotube consists of the absolutely continuous spectrum and eigenvalues of infinite multiplicities [11, 13]. Furthermore, we see other affluent results of this type of spectrum in [17]. Pankrashkin established the example where the spectrum consists of eigenvalues of infinite multiplicities (and also another interesting example where the spectrum consists of absolutely continuous spectrum and infinite multiplicities) for the Rashaba Hamiltonians on $T_{3}$ lattice. The paper [5] also includes interesting results on spectral analysis for periodic Schrödinger operators on the model of carbon nanostructure, the graphyne. The structure of the graph corresponding to graphyne defined by Do and Kuchment consists of hexagons and rhombuses. Whereas the simplest one of zigzag nanotube made of the graphene is the degenerate zigzag nanotube, the simplest one of zigzag nanotube made of the graphyne can be the generalized degenerate zigzag nanotube. Actually, the zigzag part of hexagons and rhombuses corresponds to rings of the generalized degenerate zigzag nanotube defined below and vertical lines of hexagons correspond to lines of the generalized degenerate zigzag nanotube.

We now define a periodic metric graph which possesses one line of length 1 and three rings of length 2 in the basic period cell (see FIGURE 1). The precise definition of this graph is given as follows. We put $r_{0}=\frac{1}{\pi}, T=\frac{6}{\pi}+1, \mathcal{J}=\{1,2,3,4,5,6,7\}$ and $\mathcal{Z}=\mathbf{Z} \times \mathcal{J}$. For $(n, j) \in \mathcal{Z}$, we define the segment $\Gamma_{n, j} \subset \mathbf{R}^{2}$ as

$$
\begin{aligned}
& \Gamma_{n, 1}=\{(x, 0) \mid n T<x<n T+1\}, \\
& \Gamma_{n, 2}=\left\{(x, y) \mid \quad\left\{x-\left(n T+1+r_{0}\right)\right\}^{2}+y^{2}=r_{0}^{2}, \quad y>0\right\}, \\
& \Gamma_{n, 3}=\left\{(x, y) \mid \quad\left\{x-\left(n T+1+r_{0}\right)\right\}^{2}+y^{2}=r_{0}^{2}, \quad y<0\right\}, \\
& \Gamma_{n, 4}=\left\{(x, y) \mid \quad\left\{x-\left(n T+1+3 r_{0}\right)\right\}^{2}+y^{2}=r_{0}^{2}, \quad y>0\right\}, \\
& \Gamma_{n, 5}=\left\{(x, y) \mid \quad\left\{x-\left(n T+1+3 r_{0}\right)\right\}^{2}+y^{2}=r_{0}^{2}, \quad y<0\right\}, \\
& \Gamma_{n, 6}=\left\{(x, y) \mid \quad\left\{x-\left(n T+1+5 r_{0}\right)\right\}^{2}+y^{2}=r_{0}^{2}, \quad y>0\right\}, \\
& \Gamma_{n, 7}=\left\{(x, y) \mid \quad\left\{x-\left(n T+1+5 r_{0}\right)\right\}^{2}+y^{2}=r_{0}^{2}, \quad y<0\right\} .
\end{aligned}
$$

Let each segment $\Gamma_{n, j}$ be oriented as $x$ increases. We put $\Gamma=\cup_{(n, j) \in \mathcal{Z}} \Gamma_{n, j}$.

Next, we define periodic Schrödinger operator on $\Gamma$. To this end, we describe the defi- 


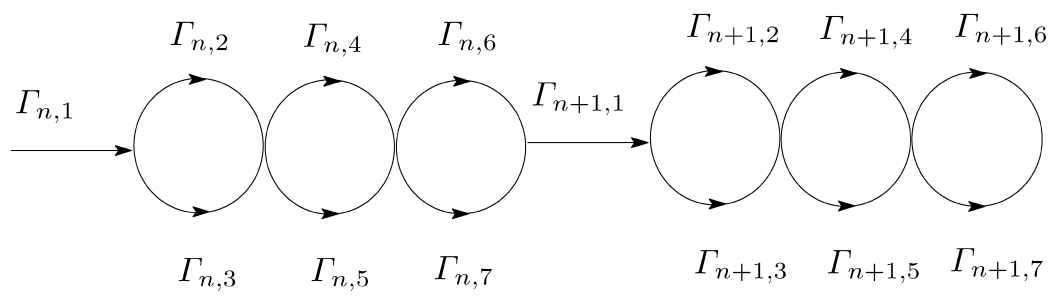

FiguRE 1. The graph $\Gamma$.

nition of the Hilbert space $L^{2}\left(\Gamma_{n, j}\right)$ for each $\alpha=(n, j) \in \mathcal{Z}$. For a function $y$ defined on $\Gamma$, we abbreviate $y_{\alpha}=\left.y\right|_{\Gamma_{\alpha}}$ for $\alpha:=(n, j) \in \mathcal{Z}$. Since the length of each segment $\Gamma_{n, j}$ is 1 , we identify each $\Gamma_{n, j}$ as the interval $(0,1)$. Owing to this identification, each $y_{\alpha}$ can be identified with a function on the interval $(0,1)$ through the local coordinate $x \in(0,1)$. Through this identification, we define the Hilbert space $L^{2}\left(\Gamma_{n, j}\right)$ as $L^{2}(0,1)$ with the standard Lebesgue measure on $(0,1)$. Moreover, we consider the Hilbert space $\mathcal{H}=\oplus_{(n, j) \in \mathcal{Z}} L^{2}\left(\Gamma_{n, j}\right)$ equipped with the inner product $\langle\psi, \varphi\rangle_{\mathcal{H}}=\sum_{\alpha \in \mathcal{Z}}\left\langle\psi_{\alpha}, \varphi_{\alpha}\right\rangle_{L^{2}\left(\Gamma_{\alpha}\right)}$ for $\psi, \varphi \in L^{2}(\Gamma)$. For $\alpha \in \mathcal{Z}$, let $f_{\alpha}^{\prime}(1)$ and $f_{\alpha}^{\prime}(0)$ imply $f_{\alpha}^{\prime}(1-0)$ and $f_{\alpha}^{\prime}(+0)$, respectively. In order to introduce the Kirchhoff vertex condition through a simple formula, we prepare notations. For $n \in \mathbf{Z}$, we put $\overline{\Gamma_{n, 1}} \cap \overline{\Gamma_{n, 2}}=\left\{X_{n, 1}\right\}, \overline{\Gamma_{n, 2}} \cap \overline{\Gamma_{n, 4}}=\left\{X_{n, 2}\right\}, \overline{\Gamma_{n, 4}} \cap \overline{\Gamma_{n, 6}}=\left\{X_{n, 3}\right\}$ and $\overline{\Gamma_{n, 6}} \cap \overline{\Gamma_{n+1,1}}=\left\{X_{n, 4}\right\}$. Furthermore, we put

$$
\begin{aligned}
& A_{1}=\left(\begin{array}{ccc}
1 & -1 & 0 \\
0 & 1 & -1 \\
0 & 0 & 0
\end{array}\right), \quad B_{1}=\left(\begin{array}{ccc}
0 & 0 & 0 \\
0 & 0 & 0 \\
-1 & 1 & 1
\end{array}\right), \\
& A_{2}=A_{3}=\left(\begin{array}{cccc}
1 & -1 & 0 & 0 \\
0 & 1 & -1 & 0 \\
0 & 0 & 1 & -1 \\
0 & 0 & 0 & 0
\end{array}\right), \quad B_{2}=B_{3}=\left(\begin{array}{cccc}
0 & 0 & 0 & 0 \\
0 & 0 & 0 & 0 \\
0 & 0 & 0 & 0 \\
-1 & -1 & 1 & 1
\end{array}\right), \\
& A_{4}=\left(\begin{array}{ccc}
1 & -1 & 0 \\
0 & 1 & -1 \\
0 & 0 & 0
\end{array}\right), \quad B_{4}=\left(\begin{array}{ccc}
0 & 0 & 0 \\
0 & 0 & 0 \\
-1 & -1 & 1
\end{array}\right), \\
& F\left(X_{n, 1}\right)=\left(\begin{array}{c}
f_{n, 1}(1) \\
f_{n, 2}(0) \\
f_{n, 3}(0)
\end{array}\right), \quad F^{\prime}\left(X_{n, 1}\right)=\left(\begin{array}{c}
f_{n, 1}^{\prime}(1) \\
f_{n, 2}^{\prime}(0) \\
f_{n, 3}^{\prime}(0)
\end{array}\right),
\end{aligned}
$$




$$
\begin{aligned}
& F\left(X_{n, 2}\right)=\left(\begin{array}{c}
f_{n, 2}(1) \\
f_{n, 3}(1) \\
f_{n, 4}(0) \\
f_{n, 5}(0)
\end{array}\right), \quad F^{\prime}\left(X_{n, 2}\right)=\left(\begin{array}{c}
f_{n, 2}^{\prime}(1) \\
f_{n, 3}^{\prime}(1) \\
f_{n, 4}^{\prime}(0) \\
f_{n, 5}^{\prime}(0)
\end{array}\right), \\
& F\left(X_{n, 3}\right)=\left(\begin{array}{c}
f_{n, 4}(1) \\
f_{n, 5}(1) \\
f_{n, 6}(0) \\
f_{n, 7}(0)
\end{array}\right), \quad F^{\prime}\left(X_{n, 3}\right)=\left(\begin{array}{c}
f_{n, 4}^{\prime}(1) \\
f_{n, 5}^{\prime}(1) \\
f_{n, 6}(0) \\
f_{n, 7}(0)
\end{array}\right), \\
& F\left(X_{n, 4}\right)=\left(\begin{array}{c}
f_{n, 6}^{\prime}(1) \\
f_{n, 6}(1) \\
f_{n, 7}(1) \\
f_{n+1,1}(0)
\end{array}\right), \quad F^{\prime}\left(X_{n, 3}\right)=\left(\begin{array}{c}
f_{n, 7}(1) \\
f_{n+1,1}^{\prime}(0)
\end{array}\right)
\end{aligned}
$$

for $n \in \mathbf{Z}$. For a real-valued function $q \in L^{2}(0,1)$, we define periodic Schrödinger operators in $\mathcal{H}$ as

$$
\begin{gathered}
\left(H f_{\alpha}\right)(x)=-f_{\alpha}^{\prime \prime}(x)+q(x) f_{\alpha}(x), \quad x \in \Gamma_{\alpha} \simeq(0,1), \quad \alpha \in \mathcal{Z}, \\
\operatorname{Dom}(H)=\left\{\bigoplus_{\alpha \in \mathcal{Z}} f_{\alpha} \in \mathcal{H} \mid \begin{array}{l}
\bigoplus_{\alpha \in \mathcal{Z}}\left(-f_{\alpha}^{\prime \prime}+q f_{\alpha}\right) \in \mathcal{H}, \\
A_{j} F\left(X_{n, j}\right)+B_{j} F^{\prime}\left(X_{n, j}\right)=O \quad \text { for } \quad(n, j) \in \mathcal{Z}
\end{array}\right\},
\end{gathered}
$$

where $O$ is the null matrix. The self-adjointness of this operator is derived in [17].

In order to analyze the spectrum of $H$, we need to review the spectral theory of the related Hill operator $H_{0}:=-d^{2} / d x^{2}+q$ in $L^{2}(\mathbf{R})$, where $q \in L^{2}(0,1)$ is extended to the 1-periodic function on $\mathbf{R}$, i.e., $q(x)$ satisfies $q(x+1)=q(x)$ for all $x \in \mathbf{R}$. According to the Floquet-Bloch theory $[6,14,20]$, we see that $\sigma\left(H_{0}\right)$ has the band structure. That is, $\sigma\left(H_{0}\right)$ is purely absolutely continuous and consists of infinitely many closed intervals whose interiors are disjoint each other. The intervals are characterized as follows. We consider the Schrödinger equation corresponding to $H_{0}$ :

$$
-y^{\prime \prime}(x, \lambda)+q(x) y(x, \lambda)=\lambda y(x, \lambda), \quad x \in \mathbf{R}, \quad \lambda \in \mathbf{C} .
$$

Let $\theta(x, \lambda)$ and $\varphi(x, \lambda)$ be the solutions to (1) subject to the initial conditions

$$
\theta(0, \lambda)=1, \quad \theta^{\prime}(0, \lambda)=0 \quad \text { and } \quad \varphi(0, \lambda)=0, \quad \varphi^{\prime}(0, \lambda)=1,
$$

respectively. Since $\theta(x, \lambda), \theta^{\prime}(x, \lambda), \varphi(x, \lambda), \varphi^{\prime}(x, \lambda)$ are entire in $\lambda \in \mathbf{C}$, so is the function $\Delta(\lambda):=\left(\theta(1, \lambda)+\varphi^{\prime}(1, \lambda)\right) / 2$. This function is called the discriminant of the spectrum of $H_{0}$ or the Lyapunov function for (1). It is known that $\Delta(\lambda)-c$ has only real simple zeroes for a fixed $c \in(-1,1)$. Moreover, the function $\Delta(\lambda) \pm 1$ has infinitely many real zeroes $\lambda_{0,0}^{+}, \lambda_{0,1}^{-}, \lambda_{0,1}^{+}, \lambda_{0,2}^{-}, \lambda_{0,2}^{+}, \ldots$, which are labeled in increasing order. We consider that 0 of $\lambda_{0, j}^{ \pm}$means that we are considering the case where there are no rings in the basic period cell. 
Then, the inequality $\lambda_{0,0}^{+}<\lambda_{0,1}^{-} \leq \lambda_{0,1}^{+}<\lambda_{0,2}^{-} \leq \lambda_{0,2}^{+}<\ldots$ holds true. The spectrum of $H_{0}$ is characterized by the Lyapunov function $\Delta(\lambda)$ and the zeroes of $\Delta(\lambda) \pm 1$ as follows:

$$
\sigma\left(H_{0}\right)=\{\lambda \in \mathbf{R}|\quad| \Delta(\lambda) \mid \leq 1\}=\bigcup_{j=1}^{\infty}\left[\lambda_{0, j-1}^{+}, \lambda_{0, j}^{-}\right] .
$$

For $j \in \mathbf{N}=\{1,2,3, \ldots\}$, the interval $B_{j}:=\left[\lambda_{0, j-1}^{+}, \lambda_{0, j}^{-}\right]$is called the $j$ th band of $\sigma\left(H_{0}\right)$. The consecutive two bands $B_{j}$ and $B_{j+1}$ are separated by the open interval $G_{j}:=\left(\lambda_{0, j}^{-}, \lambda_{0, j}^{+}\right)$. The sequence $\left\{\lambda_{0,2 j}^{+}\right\}_{j=0}^{\infty} \cup\left\{\lambda_{0,2 j}^{-}\right\}_{j=1}^{\infty}$ is the spectrum of the equation $-y^{\prime \prime}+q y=\lambda y$ satisfying the 1-periodic boundary condition: $y(x+1)=y(x)$ on $\mathbf{R}$. On the other hand, $\left\{\lambda_{0,2 j-1}^{+}\right\}_{j=1}^{\infty} \cup$ $\left\{\lambda_{0,2 j-1}^{-}\right\}_{j=1}^{\infty}$ is the spectrum of the equation $-y^{\prime \prime}+q y=\lambda y$ with the 1-anti-periodic boundary condition: $y(x+1)=-y(x)$ on $\mathbf{R}$. If there exists some $j \in \mathbf{N}$ such that $\lambda_{0, j}^{-}=\lambda_{0, j}^{+}$is valid, then the $j$ th spectral gap is degenerate, i.e., $G_{j}=\emptyset$. This implies that $B_{j}$ and $B_{j+1}$ merge, or there exists an eigenvalue, whose multiplicity is 2 , of the spectral problem $-y^{\prime \prime}+q y=\lambda y$ subject to the periodic or anti-periodic boundary conditions. Let $\sigma_{D}\left(H_{0}\right):=\left\{\mu_{n}\right\}_{n=1}^{\infty}$ be the Dirichlet spectrum, namely, the spectrum of the eigenvalue problem $-y^{\prime \prime}+q y=\lambda y$ with $y(0)=y(1)=0$, where $\left\{\mu_{n}\right\}_{n=1}^{\infty}$ is arranged in the increasing order. Then, we have $\sigma_{D}\left(H_{0}\right)=\{\lambda \in \mathbf{R} \mid \quad \varphi(1, \lambda)=0\}$ and $\mu_{n} \in\left[\lambda_{0, n}^{-}, \lambda_{0, n}^{+}\right]$for each $n \in \mathbf{N}$ (see [19]).

Let $\sigma_{\infty}(H)$ be the set of eigenvalues of $H$ with infinite multiplicities. This set is called the flat band of $H$. Defining the function

$$
D(\lambda)=8 \Delta^{4}(\lambda)+\left(-9+\theta(1, \lambda) \varphi^{\prime}(1, \lambda)\right) \Delta^{2}(\lambda)+\frac{5}{4}-\frac{\theta(1, \lambda) \varphi^{\prime}(1, \lambda)}{4}
$$

on $\mathbf{R}$ in advance, we state our theorems.

THEOREM 1. We have $\sigma(H)=\sigma_{\infty}(H) \cup \sigma_{a c}(H)$, where

$$
\sigma_{\infty}(H)=\sigma_{D}\left(H_{0}\right) \quad \text { and } \quad \sigma_{a c}(H)=\{\lambda \in \mathbf{R} \mid \quad D(\lambda) \in[-1,1]\} .
$$

In this sense, we call the function $D(\lambda)$ the discriminant of $\sigma(H)$. Next, we describe the properties of $D(\lambda)$.

THEOREM 2. (I) We have $\lim _{\lambda \rightarrow-\infty} D(\lambda)=\infty$.

(II) For $c \in\left(-\frac{649}{576}, 1\right), D(\lambda)-c$ has only real simple zeroes.

(III) The function $D^{\prime}(\lambda)$ has only real simple zeroes $\lambda_{3,1}, \lambda_{3,2}, \lambda_{3,3}, \ldots$, which are separated by the simple zeroes $\eta_{3,1}, \eta_{3,2}, \eta_{3,3}, \ldots$, of $D(\lambda)$. Namely, we have

$$
\eta_{3,1}<\lambda_{3,1}<\eta_{3,2}<\lambda_{3,2}<\eta_{3,3}<\lambda_{3,3}<\ldots .
$$

Furthermore, we have $D\left(\lambda_{3,2 n}\right) \geq 1$ and $D\left(\lambda_{3,2 n-1}\right) \leq-1$ for any $n \in \mathbf{N}$.

(IV) The function $D(\lambda)-1$ has only real zeroes. Let $z_{0}^{+}, z_{1}^{-}, z_{1}^{+}, z_{2}^{-}, z_{2}^{+}, \ldots$ be its zeroes counted with multiplicities. Then, we have

$$
z_{0}^{+}<z_{1}^{-}<z_{1}^{+}<z_{2}^{-} \leq z_{2}^{+}<z_{3}^{-}<z_{3}^{+}<z_{4}^{-} \leq z_{4}^{+}<\ldots .
$$


(v) We have

$$
\begin{aligned}
z_{0}^{+} & <x_{1}^{-}<x_{1}^{+}<z_{1}^{-}<z_{1}^{+}<x_{2}^{-}<x_{2}^{+}<z_{2}^{-} \leq z_{2}^{+} \\
& <x_{3}^{-}<x_{3}^{+}<z_{3}^{-}<z_{3}^{+}<x_{4}^{-}<x_{4}^{+}<z_{4}^{-} \leq z_{4}^{+}<\ldots,
\end{aligned}
$$

where $x_{1}^{-}, x_{1}^{+}, x_{2}^{-}, x_{2}^{+}, \ldots$ are the zeroes of $D(\lambda)+1$.

Next, we prepare notations to describe the band structure of $\sigma(H)$. Let $\lambda_{3,0}^{+}, \lambda_{3,2}^{-}, \lambda_{3,2}^{+}, \lambda_{3,4}^{-}, \lambda_{3,4}^{+}, \ldots$ be the eigenvalues, labeled in increasing order, of the operator $H_{p}$ in $\mathcal{H}$, where

$$
\begin{gathered}
\left(H_{p} f_{\alpha}\right)(x)=-f_{\alpha}^{\prime \prime}(x)+q(x) f_{\alpha}(x), \quad x \in \Gamma_{\alpha} \simeq(0,1), \quad \alpha \in \mathcal{Z}, \\
\operatorname{Dom}\left(H_{p}\right)=\left\{\begin{array}{l|l}
\bigoplus_{\alpha \in \mathcal{Z}}\left(-f_{\alpha}^{\prime \prime}+q f_{\alpha}\right) \in \mathcal{H}, \\
\bigoplus_{\alpha \in \mathcal{Z}} f_{\alpha} \in \mathcal{H}\left(X_{n, j}\right)+B_{j} F^{\prime}\left(X_{n, j}\right)=O \text { for } \quad(n, j) \in \mathcal{Z}, \\
f_{n, j}(x)=f_{n+1, j}(x) \text { and } f_{n, j}^{\prime}(x)=f_{n+1, j}^{\prime}(x), \\
\text { for }(n, j) \in \mathcal{Z} \text { and } x \in(0,1) .
\end{array}\right.
\end{gathered}
$$

On the other hand, let $\lambda_{3,1}^{-}, \lambda_{3,1}^{+}, \lambda_{3,3}^{-}, \lambda_{3,3}^{+}, \ldots$, be the spectrum of $H_{a p}$ in $\mathcal{H}$, where

$$
\begin{gathered}
\left(H_{a p} f_{\alpha}\right)(x)=-f_{\alpha}^{\prime \prime}(x)+q(x) f_{\alpha}(x), \quad x \in \Gamma_{\alpha} \simeq(0,1), \quad \alpha \in \mathcal{Z}, \\
\operatorname{Dom}\left(H_{a p}\right)=\left\{\begin{array}{l|l}
\bigoplus_{\alpha \in \mathcal{Z}}\left(-f_{\alpha}^{\prime \prime}+q f_{\alpha}\right) \in \mathcal{H}, \\
\bigoplus_{\alpha \in \mathcal{Z}} f_{\alpha} \in \mathcal{H} \mid \begin{array}{l}
A_{j} F\left(X_{n, j}\right)+B_{j} F^{\prime}\left(X_{n, j}\right)=O \quad \text { for } \quad(n, j) \in \mathcal{Z}, \\
f_{n, j}(x)=-f_{n+1, j}(x) \text { and } f_{n, j}^{\prime}(x)=-f_{n+1, j}^{\prime}(x), \\
\text { for }(n, j) \in \mathcal{Z} \text { and } x \in(0,1) .
\end{array}
\end{array}\right\} .
\end{gathered}
$$

The number 3 of $\lambda_{3, j}^{ \pm}$implies the number of rings in the basic period cell. Furthermore, we put $\alpha_{1}^{ \pm}=\arccos \sqrt{\frac{37 \mp \sqrt{73}}{72}}, \alpha_{2}^{ \pm}=\arccos \left(-\sqrt{\frac{37 \pm \sqrt{73}}{72}}\right), \alpha_{3}^{ \pm}=-\arccos \left(-\sqrt{\frac{37 \mp \sqrt{73}}{72}}\right)$, $\alpha_{4}^{ \pm}=-\arccos \sqrt{\frac{37 \pm \sqrt{73}}{72}}$. For $q \in L^{2}(0,1)$ and $n \in \mathbf{N}$, we moreover define $q_{0}=$ $\int_{0}^{1} q(x) d x, q_{s, 0, n}=\int_{0}^{1} q(x) \sin 2 n \pi x d x, \hat{q}_{n}=\int_{0}^{1} q(x) e^{2 \pi i n x} d x, u_{4 n}^{ \pm}=2 n \pi, u_{4 n-1}^{ \pm}=$ $2 n \pi-\arccos \left( \pm \frac{1}{6}\right), u_{4 n-2}^{ \pm}=2(n-1) \pi+\pi, u_{4 n-3}^{ \pm}=2(n-1) \pi+\arccos \left(\mp \frac{1}{6}\right), v_{4 n-3}^{ \pm}=$ $2(n-1) \pi+\alpha_{1}^{ \pm}, v_{4 n-2}^{ \pm}=2(n-1) \pi+\alpha_{2}^{ \pm}, v_{4 n-1}^{ \pm}=2 n \pi+\alpha_{3}^{ \pm}, v_{4 n}^{ \pm}=2 n \pi+\alpha_{4}^{ \pm}$. Under these preparations, we have the followings:

THEOREM 3. (I) We have $D\left(\lambda_{3, n}^{ \pm}\right)=(-1)^{n}$ for any $n$, and the inequality

$$
\begin{aligned}
\lambda_{3,0}^{+} & <\lambda_{3,1}^{-}<\lambda_{3,1}^{+}<\lambda_{3,2}^{-}<\lambda_{3,2}^{+}<\lambda_{3,3}^{-}<\lambda_{3,3}^{+}<\lambda_{3,4}^{-} \leq \lambda_{3,4}^{+} \\
& <\lambda_{3,5}^{-}<\lambda_{3,5}^{+}<\lambda_{3,6}^{-}<\lambda_{3,6}^{+}<\lambda_{3,7}^{-}<\lambda_{3,7}^{+}<\lambda_{3,8}^{-} \leq \lambda_{3,8}^{+}<\ldots
\end{aligned}
$$




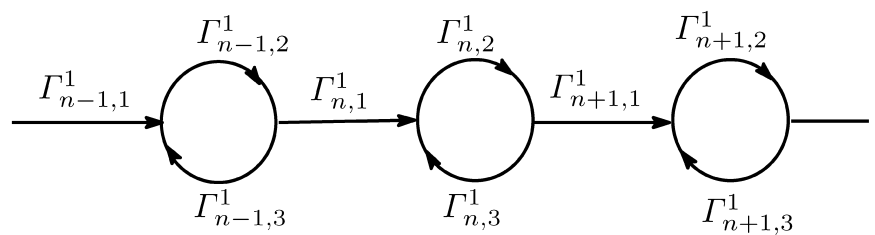

FIGURE 2. The graph $\Gamma^{1}$.

(II) The absolutely continuous spectrum of $H$ has the band structure. Namely, we have

$$
\sigma_{a c}(H)=\bigcup_{j=1}^{\infty}\left[\lambda_{3, j-1}^{+}, \lambda_{3, j}^{-}\right] .
$$

(III) For $j \in \mathbf{N}$, we define the $j$ th gap of $\sigma(H)$ by $\gamma_{j}=\left(\lambda_{3, j}^{-}, \lambda_{3, j}^{+}\right)$. Then, we have $\gamma_{4 n-3} \neq \emptyset, \gamma_{4 n-2} \neq \emptyset$ and $\gamma_{4 n-1} \neq \emptyset$ for any $n \in \mathbf{N}$.

(IV) We have the following asymptotics:

$$
\begin{aligned}
\lambda_{3,8 n-7}^{ \pm} & =\left(v_{4 n-3}^{ \pm}\right)^{2}+q_{0}+o\left(\frac{1}{n}\right), \\
\lambda_{3,8 n-6}^{ \pm} & =\left(u_{4 n-3}^{ \pm}\right)^{2}+q_{0}+o\left(\frac{1}{n}\right), \\
\lambda_{3,8 n-5}^{ \pm} & =\left(v_{4 n-2}^{ \pm}\right)^{2}+q_{0}+o\left(\frac{1}{n}\right), \\
\lambda_{3,8 n-4}^{ \pm} & =\left(u_{4 n-2}^{ \pm}\right)^{2}+q_{0} \pm \sqrt{\left|\hat{q}_{2 n-1}\right|^{2}-\frac{3}{35}\left(q_{s, 0,2 n-1}\right)^{2}}+\mathcal{O}\left(\frac{1}{n}\right), \\
\lambda_{3,8 n-3}^{ \pm} & =\left(v_{4 n-1}^{ \pm}\right)^{2}+q_{0}+o\left(\frac{1}{n}\right), \\
\lambda_{3,8 n-2}^{ \pm} & =\left(u_{4 n-1}^{ \pm}\right)^{2}+q_{0}+o\left(\frac{1}{n}\right), \\
\lambda_{3,8 n-1}^{ \pm} & =\left(v_{4 n}^{ \pm}\right)^{2}+q_{0}+o\left(\frac{1}{n}\right), \\
\lambda_{3,8 n}^{ \pm} & =\left(u_{4 n}^{ \pm}\right)^{2}+q_{0} \pm \sqrt{\left|\hat{q}_{2 n}\right|^{2}-\frac{3}{35}\left(q_{s, 0,2 n}\right)^{2}}+\mathcal{O}\left(\frac{1}{n}\right)
\end{aligned}
$$

as $n \rightarrow \infty$.

We compare our results with the classical results and related results $[11,16]$. As stated above, the spectrum of $H_{0}$ has the band structure and the band edges $\left\{\lambda_{0, n}^{ \pm}\right\}$satisfy the in- 


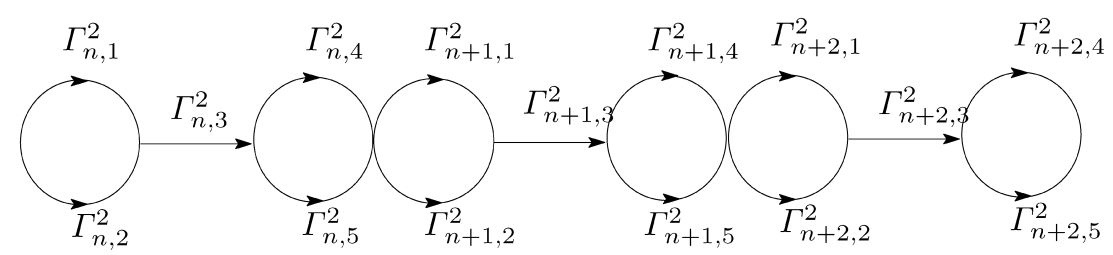

FiguRE 3. The graph $\Gamma^{2}$.

equality $\lambda_{0,0}^{+}<\lambda_{0,1}^{-} \leq \lambda_{0,1}^{+}<\lambda_{0,2}^{-} \leq \lambda_{0,2}^{+}<\ldots$ We next see the results of only the part of the degenerate zigzag nanotube established by Korotyaev and Lobanov [11]. Cutting real line $\mathbf{R}$ and adding rings of the length 1 periodically, we obtain the degenerate zigzag nanotube $\Gamma^{1}=\cup_{(n, j) \in \mathcal{Z}_{1}} \Gamma_{n, j}^{1}$ (see FIGURE 2.), where $\mathcal{J}_{1}=\{1,2,3\}$ and $\mathcal{Z}_{1}=\mathbf{Z} \times \mathcal{J}_{1}$. We shall omit the description of the precise definition of this metric graph since there may be no fear of confusion. For the operator $H_{1}$ in the Hilbert space $\mathcal{H}_{1}=L^{2}\left(\Gamma^{1}\right)=\oplus_{(n, j) \in \mathcal{Z}_{1}} L^{2}\left(\Gamma_{n, j}^{1}\right)$ defined as

$$
\begin{aligned}
& \left(H_{1} f_{n, j}\right)(x)=-f_{n, j}^{\prime \prime}(x)+q(x) f_{n, j}(x), \quad x \in(0,1) \simeq \Gamma_{n, j}^{1}, \quad(n, j) \in \mathcal{Z}_{1}, \\
& \operatorname{Dom}\left(H_{1}\right) \\
& =\left\{\begin{array}{l|l}
\bigoplus_{\alpha \in \mathcal{Z}_{1}}\left(-f_{\alpha}^{\prime \prime}+q f_{\alpha}\right) \in L^{2}\left(\Gamma^{1}\right), \\
\bigoplus_{n, j) \in \mathcal{Z}_{1}} f_{n, j} \in L^{2}\left(\Gamma^{1}\right) \mid \begin{array}{l}
f_{n, 1}^{\prime}(1)+f_{n, 2}^{\prime}(0)-f_{n, 3}^{\prime}(1)=0, \\
f_{n, 2}(0)=f_{n, 1}(1)=f_{n, 3}(1), \\
f_{n+1,1}^{\prime}(0)-f_{n, 2}^{\prime}(1)+f_{n, 3}^{\prime}(0)=0, \\
f_{n, 2}(1)=f_{n+1,1}(0)=f_{n, 3}(0) \text { for } n \in \mathbf{Z}
\end{array}
\end{array}\right.
\end{aligned}
$$

The boundary condition appearing in the $\operatorname{Dom}\left(H_{1}\right)$ is the Kirchhoff vertex condition. Korotyaev and Lobanov [11] proved that $\sigma\left(H_{1}\right)=\sigma_{\infty}\left(H_{1}\right) \cup \sigma_{a c}\left(H_{1}\right)$ and $\sigma_{a c}\left(H_{1}\right)$ has the band structure, where $\sigma_{\infty}\left(H_{1}\right)=\sigma_{D}\left(H_{0}\right)$. Designating the $j$ th band of $\sigma_{a c}\left(H_{1}\right)$ by $\left[\lambda_{1, j-1}^{+}, \lambda_{1, j}^{-}\right]$ for each $j \in \mathbf{N}$, they obtained the inequality

$$
\lambda_{1,0}^{+}<\lambda_{1,1}^{-}<\lambda_{1,1}^{+}<\lambda_{1,2}^{-} \leq \lambda_{1,2}^{+}<\lambda_{1,3}^{-}<\lambda_{1,3}^{+}<\lambda_{1,4}^{-} \leq \lambda_{1,4}^{+}<\ldots .
$$

This implies that every odd-numbered spectral gap is never degenerate, i.e., $\gamma_{1,2 n-1}:=$ $\left(\lambda_{1,2 n-1}^{-}, \lambda_{1,2 n-1}^{+}\right) \neq \varnothing$ for any $n \in \mathbf{N}$. Furthermore, let us see the result [16] in the case where we add additional rings periodically to the degenerate zigzag nanotube (see FIGURE 3). We put $\Gamma^{2}=\cup_{(n, j) \in \mathcal{Z}_{2}} \Gamma_{n, j}^{2}$, where $\mathcal{J}_{2}=\{1,2,3,4,5\}$ and $\mathcal{Z}_{2}=\mathbf{Z} \times \mathcal{J}_{2}$. We define the operator $H_{2}$ in the Hilbert space $\mathcal{H}_{2}=L^{2}\left(\Gamma^{2}\right)=\oplus_{(n, j) \in \mathcal{Z}_{2}} L^{2}\left(\Gamma_{n, j}^{2}\right)$ as

$$
\left(H_{2} f_{\alpha}\right)(x)=-f_{\alpha}^{\prime \prime}(x)+q(x) f_{\alpha}(x), \quad x \in \Gamma_{\alpha} \simeq(0,1), \quad \alpha \in \mathcal{Z}_{2},
$$

$\operatorname{Dom}\left(H_{2}\right)$ 


$$
=\left\{\begin{array}{l|l}
\bigoplus_{\alpha \in \mathcal{Z}}\left(-f_{\alpha}^{\prime \prime}+q f_{\alpha}\right) \in \mathcal{H}_{2}, \\
f_{n, 1}(1)=f_{n, 2}(1)=f_{n, 3}(0), \\
-f_{n, 1}^{\prime}(1)-f_{n, 2}^{\prime}(1)+f_{n, 3}^{\prime}(0)=0, \\
f_{n, 3}(1)=f_{n, 4}(0)=f_{n, 5}(0), \\
-f_{n, 3}^{\prime}(1)+f_{n, 4}^{\prime}(0)+f_{n, 5}^{\prime}(0)=0, \\
f_{n, 4}(1)=f_{n, 5}(1)=f_{n+1,1}(0)=f_{n+1,2}(0), \\
-f_{n, 4}^{\prime}(1)-f_{n, 5}^{\prime}(1)+f_{n+1,1}^{\prime}(0)+f_{n+1,2}^{\prime}(0)=0 \\
\text { for } n \in \mathbf{Z}
\end{array}\right\} .
$$

The boundary condition appearing in the $\operatorname{Dom}\left(H_{2}\right)$ is also the Kirchhoff vertex condition. In this case, we see that $\sigma\left(H_{2}\right)=\sigma_{\infty}\left(H_{2}\right) \cup \sigma_{a c}\left(H_{2}\right), \sigma_{\infty}\left(H_{2}\right)=\sigma_{D}\left(H_{0}\right)$ and $\sigma_{a c}\left(H_{2}\right)$ consists of infinitely many closed intervals. Let $\left[\lambda_{2, j-1}^{+}, \lambda_{2, j}^{-}\right]$be the $j$ th band of $\sigma_{a c}\left(H_{2}\right)$ for $j \in \mathbf{N}$. Then, the edges $\left\{\lambda_{2, j}^{ \pm}\right\}$satisfies the inequality

$$
\begin{aligned}
\lambda_{2,0}^{+} & <\lambda_{2,1}^{-}<\lambda_{2,1}^{+}<\lambda_{2,2}^{-}<\lambda_{2,2}^{+}<\lambda_{2,3}^{-} \leq \lambda_{2,3}^{+} \\
& <\lambda_{2,4}^{-}<\lambda_{2,4}^{+}<\lambda_{2,5}^{-}<\lambda_{2,5}^{+}<\lambda_{2,6}^{-} \leq \lambda_{2,6}^{+}<\ldots
\end{aligned}
$$

It follows by this inequality that $\gamma_{3 n-2} \neq \emptyset$ and $\gamma_{3 n-1} \neq \varnothing$ for any $n \in \mathbf{N}$, where $\gamma_{j}=$ $\left(\lambda_{2, j}^{-}, \lambda_{2, j}^{+}\right)$is the $j$ th gap of $\sigma_{a c}\left(H_{2}\right)$. These results are worthful to compare the result in Theorem 3 (III). From earlier results, we see that adding rings to the real line closely relate to the existence of non-degenerate spectral gaps. In order to show the results established by Korotyaev and Lobanov, it is necessary to solve quadratic equations on $\Delta(\lambda)$. In order to show the result [16], it is necessary to solve cubic equations on $\Delta(\lambda)$. On the other hand, we need to solve quartic equations on $\Delta(\lambda)$ in this paper because $D(\lambda)$ is quartic on $\Delta(\lambda)$. However, we see that this is not difficult because $D(\lambda)$ does not include the term of $\Delta^{3}(\lambda)$ and $\Delta(\lambda)$. In this sense, it seems to be difficult to consider the generalized degenerate zigzag nanotube which possesses 4 rings and 1 line in the basic period cell because we have to solve quintic equations. The role of this paper is to make sure that the inequality in Theorem 3 (I) holds true and that we can generalize the results $[11,16]$ when we add one ring periodically into the metric graph $\Gamma^{2}$.

In the next section, we prove that $\sigma_{a c}(H)$ has the band structure including the sense of the flat band. Namely, we prove Theorem 1.1 and 1.2. In the 3rd section, we examine the asymptotic behavior of the band edges and give the proof of Theorem 1.3.

\section{Proof of Theorem 1.1 and $\mathbf{1 . 2}$}

First, we give the proof of Theorem 1.1.

Proof of THEOREM 1.1. We pick $\lambda \in \sigma_{D}\left(H_{0}\right)$, arbitrarily. We construct infinitely many linearly independent eigenfunctions corresponding to $\lambda$. Putting $\Psi_{0,2}(x, \lambda)=\varphi(x, \lambda)$, $\Psi_{0,3}(x, \lambda)=-\varphi(x, \lambda)$ and $\Psi_{n, j}(x, \lambda)=0$ for $(n, j) \in \mathcal{Z} \backslash\{(0,2),(0,3)\}$, we define $\Psi_{n}=$ 


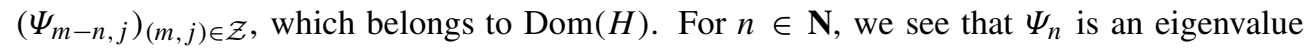
corresponding to $\lambda$. Thus, we have $\sigma_{D}\left(H_{0}\right) \subset \sigma_{\infty}$.

We next find the spectrum of $H$ besides $\sigma\left(H_{0}\right)$. We utilize a direct integral decomposition for $H$ (see $[7,20]$ ). For $\mu \in[0,2 \pi)$, we define a fiber operator $H_{\mu}$ in the Hilbert space $\mathcal{H}_{\mu}=\oplus_{j=1}^{7} L^{2}\left(\Gamma_{0, j}\right)$ as follows:

$$
\begin{gathered}
\left(H_{\mu} f_{j}\right)(x)=-f_{j}^{\prime \prime}(x)+q(x) f_{j}(x), \quad x \in \Gamma_{0, j} \simeq(0,1), \quad j \in \mathcal{J}, \\
\operatorname{Dom}\left(H_{\mu}\right)=\left\{\begin{array}{l|l}
\bigoplus_{j=1}^{7}\left(-f_{j}^{\prime \prime}+q f_{j}\right) \in \mathcal{H}_{\mu}, \\
f_{1}(1)=f_{2}(1)=f_{3}(0), \\
-f_{1}^{\prime}(1)+f_{2}^{\prime}(1)+f_{3}^{\prime}(0)=0, \\
f_{2}(1)=f_{3}(1)=f_{4}(0)=f_{5}(0), \\
-f_{2}^{\prime}(1)-f_{3}^{\prime}(1)+f_{4}^{\prime}(0)+f_{5}^{\prime}(0)=0, \\
\bigoplus_{4}(1)=f_{5}(1)=f_{6}(0)=f_{7}(0), \\
-f_{4}^{\prime}(1)-f_{5}^{\prime}(1)+f_{6}^{\prime}(0)+f_{7}^{\prime}(0)=0, \\
f_{6}(1)=f_{7}(1)=e^{i \mu} f_{1}(0), \\
-f_{6}^{\prime}(1)-f_{7}^{\prime}(1)+e^{i \mu} f_{1}^{\prime}(0)=0 .
\end{array}\right.
\end{gathered}
$$

Considering the Hilbert space

$$
\mathcal{H}=\int_{[0,2 \pi)}^{\oplus} \mathcal{H}_{\mu} \frac{d \mu}{2 \pi}=L^{2}\left([0,2 \pi), \mathcal{H}_{\mu}, \frac{d \mu}{2 \pi}\right)
$$

and the unitary operator $U: L^{2}(\Gamma) \rightarrow \mathcal{H}$ defined as

$$
(U f)(\mu)=\sum_{n \in \mathbf{Z}} e^{i n \mu} f_{n}, \quad f=\left(f_{n}\right)_{n \in \mathbf{Z}}=\left(f_{n, j}\right)_{(n, j) \in \mathcal{Z}_{1}} \in L^{2}(\Gamma),
$$

we have the direct integral representation of $H$ :

$$
U H U^{-1}=\int_{[0,2 \pi)}^{\oplus} H(\mu) \frac{d \mu}{2 \pi} .
$$

Since $H(\mu)$ acts on the finite graph $\cup_{j=1}^{7} \Gamma_{0, j}$, the spectrum of $H(\mu)$ is discrete spectrum. For $\mu \in[0,2 \pi)$, let $\left\{E_{n}(\mu)\right\}_{n \in \mathbf{N}}$ stand for the increasing sequence of the eigenvalues of $H(\mu)$, which is counted with multiplicities. Let $\mathcal{N}$ be the set of natural numbers $n$ such that $E_{n}(\mu)$ does depend on $\mu \in[0,2 \pi)$. Then, we have $\sigma(H)=\sigma_{\infty}(H) \cup \sigma_{a c}(H)$, where $\sigma_{\infty}(H)=\left\{E_{n}(\mu) \mid \quad E_{n}(\mu)\right.$ is independent of $\left.\mu \in[0,2 \pi)\right\}$ and

$$
\sigma_{a c}(H)=\bigcup_{n \in \mathcal{N}} \bigcup_{\mu \in[0,2 \pi)}\left\{E_{n}(\mu)\right\} .
$$

Since $\sigma_{D}\left(H_{0}\right) \subset \sigma_{\infty}$, we next examine $\sigma(H) \backslash \sigma_{D}\left(H_{0}\right)$. We pick $\lambda \notin \sigma_{D}\left(H_{0}\right)$, arbitrarily. We stress that $\varphi(1, \lambda) \neq 0$. In order to investigate the part of $\sigma(H) \backslash \sigma_{D}\left(H_{0}\right)$, we consider the 
characteristic equation $H_{\mu} f=\lambda f$ for $0 \neq f \in \operatorname{Dom}\left(H_{\mu}\right)$, that is, we consider the following system consisted of 9 equations:

$$
\begin{aligned}
& -f_{j}^{\prime \prime}(x)+q(x) f_{j}(x)=\lambda f_{j}(x), \quad x \in(0,1), \quad j \in \mathcal{J}, \\
& f_{1}(1)=f_{2}(1)=f_{3}(0), \\
& -f_{1}^{\prime}(1)+f_{2}^{\prime}(1)+f_{3}^{\prime}(0)=0 \\
& f_{2}(1)=f_{3}(1)=f_{4}(0)=f_{5}(0), \\
& -f_{2}^{\prime}(1)-f_{3}^{\prime}(1)+f_{4}^{\prime}(0)+f_{5}^{\prime}(0)=0 \\
& f_{4}(1)=f_{5}(1)=f_{6}(0)=f_{7}(0), \\
& -f_{4}^{\prime}(1)-f_{5}^{\prime}(1)+f_{6}^{\prime}(0)+f_{7}^{\prime}(0)=0 . \\
& f_{6}(1)=f_{7}(1)=e^{i \mu} f_{1}(0) \\
& -f_{6}^{\prime}(1)-f_{7}^{\prime}(1)+e^{i \mu} f_{1}^{\prime}(0)=0 .
\end{aligned}
$$

For fundamental solutions $\theta(x, \lambda)$ and $\varphi(x, \lambda)$, we put

$$
w(x, \lambda)=\theta(x, \lambda)-\frac{\theta(1, \lambda)}{\varphi(1, \lambda)} \varphi(x, \lambda) .
$$

Then, any solution $y$ to (1) is given by

$$
y(x, \lambda)=w(x, \lambda) y(0, \lambda)+\frac{\varphi(x, \lambda)}{\varphi(1, \lambda)} y(1, \lambda) .
$$

We put $X_{1}=f_{1}(0), X_{2}=f_{1}(1), X_{3}=f_{2}(1)$ and $X_{4}=f_{4}(1)$. Then, we obtain the followings by using (12), (14), (16) and (18):

$$
\begin{aligned}
& f_{1}(x)=w(x, \lambda) X_{1}+\frac{\varphi(x, \lambda)}{\varphi(1, \lambda)} X_{2}, \\
& f_{2}(x)=f_{3}(x)=w(x, \lambda) X_{2}+\frac{\varphi(x, \lambda)}{\varphi(1, \lambda)} X_{3}, \\
& f_{4}(x)=f_{5}(x)=w(x, \lambda) X_{3}+\frac{\varphi(x, \lambda)}{\varphi(1, \lambda)} X_{4}, \\
& f_{6}(x)=f_{7}(x)=w(x, \lambda) X_{4}+\frac{\varphi(x, \lambda)}{\varphi(1, \lambda)} e^{i \mu} X_{1} .
\end{aligned}
$$

Substituting these into (13), (15), (17) and (19), we have

$$
\begin{aligned}
& X_{1}-\left(\varphi^{\prime}(1, \lambda)+2 \theta(1, \lambda)\right) X_{2}+2 X_{3}=0, \\
& X_{2}-\left(\theta(1, \lambda)+\varphi^{\prime}(1, \lambda)\right) X_{3}+X_{4}=0, \\
& X_{3}-\left(\theta(1, \lambda)+\varphi^{\prime}(1, \lambda)\right) X_{4}+e^{i \mu} X_{1}=0, \\
& 2 X_{4}-\left(2 \varphi^{\prime}(1, \lambda)+\theta(1, \lambda)\right) e^{i \mu} X_{1}+e^{i \mu} X_{2}=0 .
\end{aligned}
$$


Since $\theta(1, \lambda)+\varphi^{\prime}(1, \lambda)=2 \Delta(\lambda)$, we obtain a system

$$
M(\lambda, \mu)\left(\begin{array}{c}
X_{1} \\
X_{2} \\
X_{3} \\
X_{4}
\end{array}\right)=0
$$

where

$$
M(\lambda, \mu)=\left(\begin{array}{cccc}
1 & -(2 \Delta(\lambda)+\theta(1, \lambda)) & 2 & 0 \\
0 & 1 & -2 \Delta(\lambda) & 1 \\
e^{i \mu} & 0 & 1 & -2 \Delta(\lambda) \\
-\left(2 \Delta(\lambda)+\varphi^{\prime}(1, \lambda)\right) e^{i \mu} & e^{i \mu} & 0 & 2
\end{array}\right) .
$$

The characteristic equation $H_{\mu} f=\lambda f$ has a non-trivial solution $f \in \operatorname{Dom}\left(H_{\mu}\right)$ if and only if this system has a non-trivial solution. Thus, we obtain $\operatorname{det} M(\lambda, \mu)=0$. By straightforward calculations, we obtain

$$
e^{-i \mu} \operatorname{det} M(\lambda, \mu)=4 \cos \mu-32 \Delta^{4}(\lambda)+36 \Delta^{2}(\lambda)-5+\left(1-4 \Delta^{2}(\lambda)\right) \theta(1, \lambda) \varphi^{\prime}(1, \lambda) .
$$

Since $\lambda$ satisfying $\operatorname{det} M(\lambda, \mu)=0$ does depend on $\mu$, we see that $\sigma_{\infty}(H) \backslash \sigma_{D}\left(H_{0}\right)=\emptyset$ and each component of $\left\{E_{n}(\mu)\right\}_{n \in \mathcal{N}}$ solves $D(\lambda)=4 \cos \mu$. Thus, we have

$$
\sigma_{a c}(H) \backslash \sigma_{D}\left(H_{0}\right)=\{\lambda \in \mathbf{R} \mid \quad D(\lambda) \in[-1,1]\} \backslash \sigma_{D}\left(H_{0}\right) .
$$

Since $\sigma_{a c}(H)$ is a closed set, we obtain $\sigma_{a c}(H)=\{\lambda \in \mathbf{R} \mid \quad D(\lambda) \in[-1,1]\}$.

Let us study the properties of $D(\lambda)$. As stated in [19], we have

$$
\begin{aligned}
\theta(1, \lambda) & =\cos \sqrt{\lambda}+\frac{1}{2 \sqrt{\lambda}} \int_{0}^{1}(\sin \sqrt{\lambda}+\sin \sqrt{\lambda}(1-2 t)) q(t) d t+\mathcal{O}\left(\frac{e^{|\Im \sqrt{\lambda}|}}{|\lambda|}\right), \\
\varphi^{\prime}(1, \lambda) & =\cos \sqrt{\lambda}+\frac{1}{2 \sqrt{\lambda}} \int_{0}^{1}(\sin \sqrt{\lambda}+\sin \sqrt{\lambda}(1-2 t)) q(t) d t+\mathcal{O}\left(\frac{e^{|\Im \sqrt{\lambda}|}}{|\lambda|}\right)
\end{aligned}
$$

as $|\lambda| \rightarrow \infty$ and hence

$$
\Delta(\lambda)=\cos \sqrt{\lambda}+\frac{q_{0} \sin \sqrt{\lambda}}{2 \sqrt{\lambda}}+\frac{S(\lambda)}{2 \sqrt{\lambda}}+\mathcal{O}\left(\frac{e^{|\Im \sqrt{\lambda}|}}{|\lambda|}\right)
$$

as $|\lambda| \rightarrow \infty$, where $\Im z$ stands for the imaginary part of a complex number $z$ and

$$
S(\lambda)=\int_{0}^{1} \sin \sqrt{\lambda}(1-2 t) q(t) d t
$$

Note that $S(\lambda) \rightarrow 0$ as $|\lambda| \rightarrow \infty$. Substituting (20), (21) and (22) into $D(\lambda)$, we obtain

$$
D(\lambda)=D_{0}(\lambda)+\frac{\mathcal{O}\left(e^{4 \mid \Im \sqrt{\lambda \mid}}\right)}{|\lambda|^{1 / 2}} \quad \text { as } \quad|\lambda| \rightarrow \infty,
$$


where $D_{0}(\lambda)=9 \cos ^{4} \sqrt{\lambda}-\frac{37}{4} \cos ^{2} \sqrt{\lambda}+\frac{5}{4}$ is the discriminant in the case where $q \equiv 0$.

ProOF OF THEOREM 1.2 (I). The asymptotics (23) gives us the proof of the statement (I) of Theorem 1.2.

Our first lemma is on the zeroes of $D(\lambda)-1$ and $D(\lambda)+\frac{649}{576}$. To describe the results, we prepare notations. For $a \in \mathbf{C}$ and $r>0$, we put

$$
\Omega(a, r)=\{\lambda \in \mathbf{C}|\quad| \sqrt{\lambda}-a \mid<r\} \quad \text { and } \quad C(a, r)=\{\lambda \in \mathbf{C}|\quad| \sqrt{\lambda}-a \mid=r\} .
$$

Moreover, we define $C(r)=C_{1}(r)-C_{2}(r)-C_{3}(r)+C_{4}(r)$, where

$$
\begin{aligned}
& C_{1}(r)=\{\lambda \in \mathbf{C} \mid \quad \sqrt{\lambda}=r+i r t, \quad-1 \leq t \leq 1\}, \\
& C_{2}(r)=\{\lambda \in \mathbf{C} \mid \quad \sqrt{\lambda}=r t+i r, \quad-1 \leq t \leq 1\}, \\
& C_{3}(r)=\{\lambda \in \mathbf{C} \mid \quad \sqrt{\lambda}=-r+i r t, \quad-1 \leq t \leq 1\}, \\
& C_{4}(r)=\{\lambda \in \mathbf{C} \mid \quad \sqrt{\lambda}=r t-i r, \quad-1 \leq t \leq 1\}
\end{aligned}
$$

for $r>0$. Let $\tilde{\Omega}(r)$ be the region surrounded by $C(r)$.

LEMMA 1. (I) There exists some $n_{0} \in \mathbf{N}$ such that $D(\lambda)-1$ has exactly two zeroes in both $\Omega\left(n \pi, \frac{\pi}{8}\right)$ and $\Omega\left(n \pi+\frac{\pi}{2}, \frac{\pi}{8}\right)$ and $8 n-1$ zeroes in $\tilde{\Omega}\left(2 n \pi-\frac{\pi}{8}\right)$ for any $n \geq n_{0}$, counted with multiplicities. There are no other zeroes.

(II) There exists some $n_{0} \in \mathbf{N}$ such that $D(\lambda)+\frac{649}{576}$ has exactly two zeroes in both $\Omega\left(n \pi+\frac{\pi}{4}, \frac{\pi}{8}\right)$ and $\Omega\left(n \pi+\frac{3 \pi}{4}, \frac{\pi}{8}\right)$ and $8 n$ zeroes in $\tilde{\Omega}\left(2 n \pi-\frac{\pi}{8}\right)$ for any $n \geq n_{0}$, counted with multiplicities. There are no other zeroes.

Proof. We put $\sqrt{\lambda}=n \pi+\alpha+i \beta$, where $\alpha=\frac{\pi}{8} \cos \theta, \beta=\frac{\pi}{8} \sin \theta$ and $\theta=[0,2 \pi]$. We note that

$$
D_{0}(\lambda)-1=9(\cos \sqrt{\lambda}+1)(\cos \sqrt{\lambda}-1)\left(\cos \sqrt{\lambda}+\frac{1}{6}\right)\left(\cos \sqrt{\lambda}-\frac{1}{6}\right) .
$$

For $p=1,-1, \frac{1}{6},-\frac{1}{6}$, we claim that there exists some constant $C_{p}>0$ satisfying $\mid \cos \sqrt{\lambda}+$ $p \mid \geq C_{p}$. It follows by a straightforward calculation that

$$
|\cos \sqrt{\lambda}+p|^{2}=A(\theta)^{2}+B(\theta)^{2},
$$

where $A(\theta)=(-1)^{n} \cos \alpha \cosh \beta+p$ and $B(\theta)=\sin \alpha \sinh \beta$. Let us show that $(A(\theta), B(\theta))=(0,0)$ does not hold true for any $\theta$. We first consider the case where $\sin \alpha=0$, which implies $\theta=\frac{\pi}{2}, \frac{3}{2} \pi$. If $\theta=\frac{\pi}{2}$, then we have $\beta=\frac{\pi}{8}$ and hence $A\left(\frac{\pi}{2}\right)=(-1)^{n} \cosh \frac{\pi}{8}+p \neq 0$. If $\theta=\frac{3}{2} \pi$, then we have $\beta=-\frac{\pi}{8}$ and hence $A\left(\frac{3}{2} \pi\right)=(-1)^{n} \cosh \frac{\pi}{8}+p \neq 0$. On the other hand, we consider the case where $\sinh \beta=0$, which implies $\beta=0$. Then, we see that $\theta=0, \pi, 2 \pi$. If $\theta=0,2 \pi$, then we have $\alpha=\frac{\pi}{8}$ and hence $A(\theta)=\cos \frac{\pi}{8}+p \neq 0$. If $\theta=\pi$, then we obtain $\alpha=-\frac{\pi}{8}$ and 
hence $A(\theta)=\cos \frac{\pi}{8}+p \neq 0$. Since $|\cos \sqrt{\lambda}+p|^{2}$ is continuous in $\theta \in[0,2 \pi]$, there exists its minimum. The minimum is strictly positive because there does not exist $\theta$ satisfying $(A(\theta), B(\theta))=(0,0)$. Thus, there exists some constant $C_{p}>0$ satisfying $|\cos \sqrt{\lambda}+p| \geq C_{p}$. This combined with (24) means that there exists some constant $C>0$ satisfying $\left|D_{0}(\lambda)-1\right| \geq C$ on $C\left(n \pi, \frac{\pi}{8}\right)$. Similarly, we see that there exists some $C>0$ such that $\left|D_{0}(\lambda)-1\right| \geq C$ on $\Omega\left(n \pi+\frac{\pi}{2}, \frac{\pi}{8}\right)$. So, we see that

$$
\frac{\left|(D(\lambda)-1)-\left(D_{0}(\lambda)-1\right)\right|}{\left|D_{0}(\lambda)-1\right|} \leq \mathcal{O}\left(\frac{1}{n}\right) \quad \text { as } n \rightarrow \infty
$$

on both $C\left(n \pi, \frac{\pi}{8}\right)$ and $C\left(n \pi+\frac{\pi}{2}, \frac{\pi}{8}\right)$ due to (23). This combined with Rouché's theorem means that the number of zeroes of $D(\lambda)-1$ and $D_{0}(\lambda)-1$ inside both $\Omega\left(n \pi, \frac{\pi}{8}\right)$ and $\Omega\left(n \pi+\frac{\pi}{2}, \frac{\pi}{8}\right)$ are the same for a large $n \in \mathbf{N}$. Let us show that $D_{0}(\lambda)-1$ has exactly two zeroes in both $\Omega\left(n \pi, \frac{\pi}{8}\right)$ and $\Omega\left(n \pi+\frac{\pi}{2}, \frac{\pi}{8}\right)$. We recall (24), which implies that $D_{0}(\lambda)-1=0$ is equivalent to $\cos \sqrt{\lambda}= \pm 1, \pm \frac{1}{6}$. Since $\frac{3}{8} \pi<\arccos \frac{1}{6}<\frac{\pi}{2}$ and $\frac{\pi}{2}<\arccos \left(-\frac{1}{6}\right)<\frac{5}{8} \pi$ because of $\cos ^{2} \frac{3}{8} \pi=\left(1+\cos \frac{3}{4} \pi\right) / 2>\frac{1}{36}$, we see that $\Omega\left(n \pi+\frac{\pi}{2}, \frac{\pi}{8}\right)$ includes exactly two zeroes of $D_{0}(\lambda)-1$, counted with multiplicities. On the other hand, since the multiplicity of both $\cos \sqrt{\lambda}=1$ and $\cos \sqrt{\lambda}=-1$ is 2 , we see that $\Omega\left(n \pi, \frac{\pi}{8}\right)$ includes 2 zeroes of $D_{0}(\lambda)-1$, counted with multiplicities. Thus, we see that $D(\lambda)-1$ has exactly two zeroes, counted with multiplicities, in both $\Omega\left(n \pi, \frac{\pi}{8}\right)$ and $\Omega\left(n \pi+\frac{\pi}{2}, \frac{\pi}{8}\right)$ for a large $n \in \mathbf{N}$.

We next show that $D(\lambda)-1$ has exactly $8 n-1$ zeroes in $\tilde{\Omega}\left(2 n \pi-\frac{\pi}{8}\right)$ for a large $n \in \mathbf{N}$. We claim that for $p= \pm 1, \pm \frac{1}{6}$, there exists some $M_{p}>0$ such that

$$
e^{\mid \Im \sqrt{\lambda \mid}}<M_{p}|\cos \sqrt{\lambda}+p|
$$

on $C_{1}\left(2 n \pi-\frac{\pi}{8}\right), C_{2}\left(2 n \pi-\frac{\pi}{8}\right), C_{3}\left(2 n \pi-\frac{\pi}{8}\right)$ and $C_{4}\left(2 n \pi-\frac{\pi}{8}\right)$. We first consider $\lambda \in$ $C_{1}\left(2 n \pi-\frac{\pi}{8}\right)$. Putting $\sqrt{\lambda}=2 n \pi-\frac{\pi}{8}+i\left(2 n \pi-\frac{\pi}{8}\right) t$ and $-1 \leq t \leq 1$, we have

$|\cos \sqrt{\lambda}+p|^{2}=\left(\cos \left(2 n \pi-\frac{\pi}{8}\right) \cosh \left(2 n \pi-\frac{\pi}{8}\right) t+p\right)^{2}+\sin ^{2}\left(2 n \pi-\frac{\pi}{8}\right) \sinh ^{2}\left(2 n \pi-\frac{\pi}{8}\right) t$.

So, we see that

$$
\frac{e^{2 \mid \Im \sqrt{\lambda \mid}}}{|\cos \sqrt{\lambda}+p|^{2}}=\frac{e^{2\left|2 n \pi-\frac{\pi}{8}\right| t}}{\left(\cos \frac{\pi}{8} \cosh \left(2 n \pi-\frac{\pi}{8}\right) t+p\right)^{2}+\sin ^{2} \frac{\pi}{8} \sinh ^{2}\left(2 n \pi-\frac{\pi}{8}\right) t} .
$$

We put

$$
f(x)=\frac{e^{2 x}}{\left(\cos \frac{\pi}{8} \cosh x+p\right)^{2}+\sin ^{2} \frac{\pi}{8} \sinh ^{2} x}
$$

and $g(x)=\left(\cos \frac{\pi}{8} \cosh x+p\right)^{2}+\sin ^{2} \frac{\pi}{8} \sinh ^{2} x$. We show that there exists some $\tilde{C}_{p}>0$ such that $g(x) \geq \tilde{C}_{p}$. We see that $\sinh x=0$ and $\cos \frac{\pi}{8} \cosh x+p=0$ do not hold true 
simultaneously. In addition, $g(x) \rightarrow \infty$ as $x \rightarrow \pm \infty$. So, we see that there exists some $\tilde{C}_{p}>0$ such that $g(x) \geq \tilde{C}_{p}$. Since $g(x)$ is continuous and $g(x) \neq 0$ on $\mathbf{R}, f(x)$ is continuous on $\mathbf{R}$. Furthermore, we see $f(x) \rightarrow 4$ as $x \rightarrow \infty$ and $f(x) \rightarrow 0$ as $x \rightarrow-\infty$. Thus, we notice that there exists some $\check{C}_{p}>0$ such that $f(x) \leq \check{C}_{p}$. This combined with (25) means that there exists some $M_{p}>0$ such that $e^{|\Im \sqrt{\lambda}|}<M_{p}|\cos \sqrt{\lambda}+p|$ on $C_{1}\left(2 n \pi-\frac{\pi}{8}\right)$. Similarly, we obtain the same inequality on $C_{3}\left(2 n \pi-\frac{\pi}{8}\right)$.

We next pick $\lambda \in C_{2}\left(2 n \pi-\frac{\pi}{8}\right)$, arbitrarily. Putting $2 n \pi-\frac{\pi}{8}=m,-1 \leq t \leq 1$ and $\sqrt{\lambda}=m t+i m$, we have $|\cos \sqrt{\lambda}+p|^{2}=(\cos m t \cosh m+p)^{2}+\sin ^{2} m t \sinh ^{2} m$. Putting

$$
g_{m}(t)=\left(\cos m t \cdot \frac{\cosh m}{e^{m}}+\frac{p}{e^{m}}\right)^{2}+\sin ^{2} m t \cdot \frac{\sinh ^{2} m}{e^{2 m}},
$$

we have

$$
\frac{e^{2|\Im \sqrt{\lambda}|}}{|\cos \sqrt{\lambda}+p|^{2}}=\frac{1}{g_{m}(t)} \leq\left|\frac{1}{g_{m}(t)}-4\right|+4=\frac{4}{g_{m}(t)}\left|\frac{1}{4}-g_{m}(t)\right|+4 .
$$

We obtain

$$
\begin{aligned}
& g_{m}(t) \\
= & \frac{e^{2 m}+2+e^{-2 m}}{4 e^{2 m}} \cos ^{2} m t+2 p \frac{e^{m}+e^{-m}}{2 e^{2 m}} \cos m t+\frac{p^{2}}{e^{2 m}}+\sin ^{2} m t \frac{e^{2 m}-2+e^{-2 m}}{4 e^{2 m}} \\
= & \frac{e^{2 m}+e^{-2 m}}{4 e^{2 m}}+\frac{1}{2 e^{2 m}} \cos 2 m t+p \frac{e^{m}+e^{-m}}{e^{2 m}} \cos m t+\frac{p^{2}}{e^{2 m}} \\
\geq & \frac{e^{2 m}+e^{-2 m}}{4 e^{2 m}}-\frac{1}{2 e^{2 m}}-|p| \frac{e^{m}+e^{-m}}{e^{2 m}}+\frac{p^{2}}{e^{2 m}} \\
= & \frac{\cosh ^{2} m-1-2|p| \cosh m+|p|^{2}}{e^{2 m}} \\
= & \frac{(\cosh m-|p|)^{2}-1}{e^{2 m}} .
\end{aligned}
$$

Putting $h(x)=\frac{e^{2 x}}{(\cosh x-|p|)^{2}-1}$, we have

$$
h^{\prime}(x)=\frac{-\frac{5}{2}|p| e^{3 x}+o\left(e^{3 x}\right)}{(\cosh x-|p|)^{2}-1} \quad \text { as } \quad x \rightarrow \infty .
$$

This implies that there exists some $x_{0}(p) \in \mathbf{R}$ such that $h^{\prime}(x)<0$ for $x>x_{0}(p)$. Namely, if $x>x_{0}(p)$, then we have $h(x)<h\left(x_{0}(p)\right)$. That is, there exists some $m_{0}(p) \in \mathbf{N}$ such that

$$
g_{m}(t) \geq \frac{\left(\cosh m_{0}(p)-|p|\right)^{2}-1}{e^{2 m_{0}(p)}}>0
$$


for $m>m_{0}(p)$. Since

$$
\begin{aligned}
\left|g_{m}(t)-\frac{1}{4}\right| & =\left|\frac{1}{4} e^{-4 m}+\frac{1}{2 e^{2 m}} \cos 2 m t+2 p \cos m t \frac{\cosh m}{e^{2 m}}+\frac{p^{2}}{e^{2 m}}\right| \\
& \leq \frac{1}{4}+\frac{1}{2}+2|p|+|p|^{2},
\end{aligned}
$$

it follows by (26) and (27) that there exists some $M_{p}>0$ such that $e^{\mid \Im \sqrt{\lambda \mid}}<M_{p}|\cos \sqrt{\lambda}+p|$ on $C_{2}\left(2 n \pi-\frac{\pi}{8}\right)$ for a large $n \in \mathbf{N}$. Similarly, we obtain the same inequality on $C_{2}\left(2 n \pi-\frac{\pi}{8}\right)$ if $n \in \mathbf{N}$ is enough large. Thus, (23) and (24) imply that

$$
\left|(D(\lambda)-1)-\left(D_{0}(\lambda)-1\right)\right|=\left|D_{0}(\lambda)-1\right| \cdot \mathcal{O}\left(\frac{1}{n}\right)
$$

on $C\left(2 n \pi-\frac{\pi}{8}\right)$ for a large $n \in \mathbf{N}$. Since $D_{0}(\lambda)-1$ has exactly $8 n-1$ zeroes inside $\Omega\left(2 n \pi-\frac{\pi}{8}\right)$, it follows by Rouché's theorem that $D(\lambda)-1$ also has exactly $8 n-1$ zeroes inside $\Omega\left(2 n \pi-\frac{\pi}{8}\right)$ if $n \in \mathbf{N}$ is enough large.

Noting that $D_{0}(\lambda)+\frac{649}{576}=9\left(\cos ^{2} \sqrt{\lambda}-\frac{37}{72}\right)^{2}$, we also get the statement (II).

Our next goal is to show that $D(\lambda)-c$ has only simple real zeroes for a fixed $c \in$ $\left(-\frac{649}{576}, 1\right)$. We first examine the zeroes of $D_{0}(\lambda)-c=9 \cos ^{4} \sqrt{\lambda}-\frac{37}{4} \cos ^{2} \sqrt{\lambda}+\frac{5}{4}-c$. We see that $D_{0}(\lambda)-c=0$ is equivalent to

$$
\begin{gathered}
\cos \sqrt{\lambda}=\sqrt{\frac{37+\sqrt{649+576 c}}{72}} \in\left(\sqrt{\frac{37}{72}}, 1\right), \quad-\sqrt{\frac{37+\sqrt{649+576 c}}{72}} \in\left(-1,-\sqrt{\frac{37}{72}}\right), \\
\sqrt{\frac{37-\sqrt{649+576 c}}{72}} \in\left(\frac{1}{6}, \sqrt{\frac{37}{72}}\right), \quad-\sqrt{\frac{37-\sqrt{649+576 c}}{72}} \in\left(-\sqrt{\frac{37}{72}},-\frac{1}{6}\right) .
\end{gathered}
$$

For a fixed $c \in\left(-\frac{649}{576}, 1\right), D_{0}(\lambda)-c$ has 8 zeroes in $(2 n \pi, 2(n+1) \pi)$ for each $n \in \mathbf{N}$. For a fixed $c \in\left(-\frac{649}{576}, 1\right)$, we put

$$
\begin{aligned}
& \alpha_{1}(c)=\arccos \sqrt{\frac{37+\sqrt{649+576 c}}{72}} \in\left(0, \arccos \sqrt{\frac{37}{72}}\right), \\
& \alpha_{2}(c)=\arccos \sqrt{\frac{37-\sqrt{649+576 c}}{72}} \in\left(\arccos \sqrt{\left.\frac{37}{72}, \arccos \frac{1}{6}\right),}\right. \\
& \alpha_{3}(c)=\arccos \left(-\sqrt{\frac{37-\sqrt{649+576 c}}{72}}\right) \in\left(\arccos \left(-\frac{1}{6}\right), \arccos \left(-\sqrt{\frac{37}{72}}\right),\right.
\end{aligned}
$$




$$
\alpha_{4}(c)=\arccos \left(-\sqrt{\frac{37+\sqrt{649+576 c}}{72}}\right) \in\left(\arccos \left(-\sqrt{\frac{37}{72}}\right), \pi\right) .
$$

For any $c \in\left(-\frac{649}{576}, 1\right)$, we see that $\alpha_{1}(c)<\alpha_{2}(c)<\alpha_{3}(c)<\alpha_{4}(c)$. Furthermore, $\alpha_{1}(c)$ and $\alpha_{3}(c)$ are increasing in $c \in\left(-\frac{649}{576}, 1\right)$, whereas $\alpha_{2}(c)$ and $\alpha_{4}(c)$ are decreasing in $c \in$ $\left(-\frac{649}{576}, 1\right)$. Thus, we see that $\cos \alpha_{1}(c)$ and $\cos \alpha_{3}(c)$ are increasing in $c \in\left(-\frac{649}{576}, 1\right)$, whereas $\cos \alpha_{2}(c)$ and $\cos \alpha_{4}(c)$ are decreasing in $c \in\left(-\frac{649}{576}, 1\right)$. Moreover, we see that

$$
\begin{aligned}
0 & =\alpha_{1}(1)<\alpha_{1}(c)<\alpha_{1}\left(-\frac{649}{576}\right)=\arccos \sqrt{\frac{37}{72}}=\alpha_{2}\left(-\frac{649}{576}\right)<\alpha_{2}(c) \\
& <\alpha_{2}(1)=\arccos \frac{1}{6}<\arccos \left(-\frac{1}{6}\right)<\alpha_{3}(1)<\alpha_{3}(c)<\alpha_{3}\left(-\frac{649}{576}\right) \\
& =\arccos \left(-\sqrt{\frac{37}{72}}\right)=\alpha_{4}\left(-\frac{649}{576}\right)<\alpha_{4}(c)<\alpha_{4}(1)=\pi
\end{aligned}
$$

for any $c \in\left(-\frac{649}{576}, 1\right)$. Putting $\alpha_{5}(c)=2 \pi-\alpha_{4}(c), \alpha_{6}(c)=2 \pi-\alpha_{3}(c), \alpha_{7}(c)=2 \pi-\alpha_{2}(c)$ and $\alpha_{8}(c)=2 \pi-\alpha_{1}(c)$, we define $\alpha_{n, j}(c)=\alpha_{j}(c)+2 n \pi$ for $j=1,2,3, \ldots, 8$ and $n \in \mathbf{N}_{0}:=\{0,1,2, \ldots\}$. Note that $\alpha_{n, j} \in(2 n \pi, 2(n+1) \pi)$.

We prepare notations to use the Rouché's theorem. Putting $\gamma_{0}=0, \gamma_{1}=\arccos \sqrt{\frac{37}{72}}$, $\gamma_{2}=\arccos \frac{1}{6}, \gamma_{3}=\arccos \left(-\frac{1}{6}\right), \gamma_{4}=\arccos \left(-\sqrt{\frac{37}{72}}\right), \gamma_{5}=\pi, \gamma_{6}=2 \pi-\gamma_{4}, \gamma_{7}=2 \pi-\gamma_{3}$, $\gamma_{8}=2 \pi-\gamma_{2}, \gamma_{9}=2 \pi-\gamma_{1}$ and $\gamma_{10}=2 \pi$, we reset notations by

$$
\begin{aligned}
& C_{1}(n)=C_{0}^{-}(n)+\tilde{C}_{1}(n)-C_{0}^{+}(n)-\tilde{C}_{0}(n), \\
& C_{2}(n)=C_{1}^{-}(n)+\tilde{C}_{2}(n)-C_{1}^{+}(n)-\tilde{C}_{1}(n), \\
& C_{3}(n)=C_{3}^{-}(n)+\tilde{C}_{4}(n)-C_{3}^{+}(n)-\tilde{C}_{3}(n), \\
& C_{4}(n)=C_{4}^{-}(n)+\tilde{C}_{5}(n)-C_{4}^{+}(n)-\tilde{C}_{4}(n), \\
& C_{5}(n)=C_{5}^{-}(n)+\tilde{C}_{6}(n)-C_{5}^{+}(n)-\tilde{C}_{5}(n), \\
& C_{6}(n)=C_{6}^{-}(n)+\tilde{C}_{7}(n)-C_{6}^{+}(n)-\tilde{C}_{6}(n), \\
& C_{7}(n)=C_{8}^{-}(n)+\tilde{C}_{9}(n)-C_{8}^{+}(n)-\tilde{C}_{8}(n), \\
& C_{8}(n)=C_{9}^{-}(n)+\tilde{C}_{10}(n)-C_{9}^{+}(n)-\tilde{C}_{9}(n),
\end{aligned}
$$

where

$$
\begin{aligned}
& \tilde{C}_{j}(n)=\left\{\lambda \in \mathbf{C} \mid \quad \sqrt{\lambda}=2 n \pi+\gamma_{j}+n i t, \quad-1 \leq t \leq 1\right\}, \\
& C_{j}^{ \pm}(n)=\left\{\lambda \in \mathbf{C} \mid \quad \sqrt{\lambda}=2 n \pi+t \pm n i, \quad \gamma_{j} \leq t \leq \gamma_{j+1}\right\}
\end{aligned}
$$


for $j=0,1,2, \ldots, 10$ and $n \in \mathbf{N}$. Furthermore, we put

$$
\begin{array}{cl}
\tilde{C}_{0}(-n)=\{\lambda \in \mathbf{C} \mid & \sqrt{\lambda}=-2 n \pi+n i t, \quad-1 \leq t \leq 1\}, \\
\tilde{C}_{0}^{ \pm}(n)=\{\lambda \in \mathbf{C} \mid & \sqrt{\lambda}= \pm n i+2 \pi t, \quad-1 \leq t \leq 1\}
\end{array}
$$

and $C_{0}(n)=\tilde{C}_{0}^{-}(n)+\tilde{C}_{0}(n)-\tilde{C}_{0}^{+}(n)-\tilde{C}_{0}(-n)$ for $n \in \mathbf{N}$. For a fixed $c \in\left(-\frac{649}{576}, 1\right)$, we see that $D_{0}(\lambda)-c$ has exactly $8 n$ zeroes in $C_{0}(n)$ and exactly one zero in $C_{j}(n)$ for each $j=1,2,3, \ldots, 8$ and $n \in \mathbf{N}$. Furthermore, we obtain the followings.

LEMMA 2. For a fixed $c \in\left(-\frac{649}{576}, 1\right)$, there exists some $n_{0} \in \mathbf{N}$ such that $D(\lambda)-c$ has exactly $8 n_{0}$ zeroes in $C_{0}\left(n_{0}\right)$ and exactly one zeroes in $C_{j}(n)$ for each $j=1,2,3, \ldots, 8$ if $n \geq n_{0}$, where the number of zeroes is counted with multiplicities. Moreover, there are no other zeroes of $D(\lambda)-c$.

PROOF. For a fixed $c \in\left(-\frac{649}{576}, 1\right)$, we put $p_{1}(c)=\sqrt{\frac{37+\sqrt{649+576 c}}{72}}, p_{2}(c)=$ $-\sqrt{\frac{37+\sqrt{649+576 c}}{72}}, p_{3}(c)=\sqrt{\frac{37-\sqrt{649+576 c}}{72}}, p_{4}(c)=-\sqrt{\frac{37-\sqrt{649+576 c}}{72}}$. We recall that

$$
D_{0}(\lambda)-c=\left(\cos \sqrt{\lambda}-p_{1}(c)\right)\left(\cos \sqrt{\lambda}-p_{2}(c)\right)\left(\cos \sqrt{\lambda}-p_{3}(c)\right)\left(\cos \sqrt{\lambda}-p_{4}(c)\right) .
$$

For $p(c)=p_{1}(c), p_{2}(c), p_{3}(c), p_{4}(c)$ and $j=0,1,2, \ldots, 8$, there exist some constants $C_{p(c), j}>0$ and $n_{0}(p(c), j) \in \mathbf{N}$ such that

$$
e^{\mid \Im \sqrt{\lambda \mid}} \leq C_{p(c), j}|\cos \sqrt{\lambda}-p(c)|
$$

on $C_{j}(n)$ if $n \geq n_{0}(p(c), j)$. This statement is shown in a similar way to Lemma 1 . It follows by (23), (28) and (29) that

$$
\left|(D(\lambda)-c)-\left(D_{0}(\lambda)-c\right)\right|=\left|D_{0}(\lambda)-c\right| \cdot \mathcal{O}\left(\frac{1}{|\lambda|^{1 / 2}}\right)
$$

on $C_{j}(n)$ if $n \geq n_{0}(p(c), j)$. Thus, it holds true for a large $n \in \mathbf{N}$ that

$$
\left|(D(\lambda)-c)-\left(D_{0}(\lambda)-c\right)\right|<\left|D_{0}(\lambda)-c\right|
$$

on $C_{j}(n)$. So, it follows by the Rouché's theorem that the numbers of $D(\lambda)-c$ and $D_{0}(\lambda)-c$ are the same for a large $n \in \mathbf{N}$. Therefore, we obtain our assertion.

In order to prove that $D(\lambda)-c$ has only simple real zeroes for a fixed $c \in\left(-\frac{649}{576}, 1\right)$, we need two more lemma.

LEMMA 3. (I) If $\lambda \in \sigma_{D}\left(H_{0}\right)$, then we have $D(\lambda) \geq 1$.

(II) If $\lambda$ satisfies that $\Delta(\lambda)=0$, then we have $D(\lambda) \geq \frac{5}{4}$.

(III) If $\lambda$ satisfies that $\Delta^{2}(\lambda)=\frac{37}{72}$, then we have $D(\lambda) \leq-\frac{649}{576}$. 
Proof. Let us show the first statement. We pick $\lambda \in \sigma_{D}\left(H_{0}\right)$, arbitrarily. Since $\varphi(1, \lambda)=0$, it follows by $\theta(1, \lambda) \varphi(1, \lambda)-\theta^{\prime}(1, \lambda) \varphi(1, \lambda)=1$ that $\theta(1, \lambda) \varphi^{\prime}(1, \lambda)=1$. Thus, we see that $D(\lambda)=8 \Delta^{4}(\lambda)-8 \Delta^{2}(\lambda)+1=8\left(\Delta^{2}(\lambda)-\frac{1}{2}\right)^{2}-1$. Since $\Delta^{2}(\lambda) \geq 1$ for $\lambda \in \sigma_{D}\left(H_{0}\right)$, we have $D(\lambda) \geq 1$.

We next show that second statement. We see that $\Delta(\lambda)=0$ implies that $\theta(1, \lambda)=$ $-\varphi^{\prime}(1, \lambda)$. Thus, we have $D(\lambda)=\frac{5}{4}+\frac{(\theta(1, \lambda))^{2}}{4} \geq \frac{5}{4}$.

We finally show the third statement. We put

$$
\Delta_{-}(\lambda)=\frac{\theta(1, \lambda)-\varphi^{\prime}(1, \lambda)}{2} .
$$

By straightforward calculations, we have

$$
\Delta^{2}(\lambda)+\Delta_{-}^{2}(\lambda)=\frac{\theta^{2}(1, \lambda)+\left(\varphi^{\prime}(1, \lambda)\right)^{2}}{2}
$$

and $4 \Delta^{2}(\lambda)=\theta^{2}(1, \lambda)+2 \theta(1, \lambda) \varphi^{\prime}(1, \lambda)+\left(\varphi^{\prime}(1, \lambda)\right)^{2}$ and hence $\theta(1, \lambda) \varphi^{\prime}(1, \lambda)=\Delta^{2}(\lambda)-$ $\Delta_{-}^{2}(\lambda)$. So, we obtain

$$
\begin{aligned}
D(\lambda) & =9 \Delta^{4}(\lambda)-\frac{37}{4} \Delta^{2}(\lambda)+\frac{5}{4}+\left(\frac{1}{4}-\Delta^{2}(\lambda)\right) \Delta_{-}^{2}(\lambda) \\
& =9\left(\Delta^{2}(\lambda)-\frac{37}{72}\right)^{2}-\frac{649}{576}+\left(\frac{1}{4}-\Delta^{2}(\lambda)\right) \Delta_{-}^{2}(\lambda) .
\end{aligned}
$$

Therefore, we see that $D(\lambda) \leq-\frac{649}{576}$ if $\Delta^{2}(\lambda)=\frac{37}{72}$.

Due to Theorem 2 (I), Lemma 3 (III) and the intermediate value theorem, there exists $\lambda \in \mathbf{R}$ such that $D(\lambda)=1$. Let $\eta_{0} \in \mathbf{R}$ be the minimum of $\lambda$ satisfying $D(\lambda)=1$. Moreover, we define $\left\{\eta_{j}^{+}\right\}_{j=1}^{\infty},\left\{\eta_{j}^{-}\right\}_{j=1}^{\infty}$ and $\left\{\eta_{j}\right\}_{j=1}^{\infty}$ be the increasing sequence satisfying

$$
\begin{gathered}
\left\{\eta_{j}^{+}\right\}_{j=1}^{\infty}=\left\{\lambda \in \mathbf{R} \mid \quad \Delta(\lambda)=\sqrt{\frac{37}{72}}\right\}, \\
\left\{\eta_{j}^{-}\right\}_{j=1}^{\infty}=\left\{\lambda \in \mathbf{R} \mid \quad \Delta(\lambda)=-\sqrt{\frac{37}{72}}\right\}, \\
\left\{\eta_{j}\right\}_{j=1}^{\infty}=\{\lambda \in \mathbf{R} \mid \quad \Delta(\lambda)=0\} .
\end{gathered}
$$

Since the behavior of $\Delta(\lambda)$ is well-known in [14, Theorem 2.1], we see that

$$
\begin{aligned}
\eta_{0} & <\eta_{1}^{+}<\eta_{1}<\eta_{1}^{-}<\mu_{1}<\eta_{2}^{-}<\eta_{2}<\eta_{2}^{+}<\mu_{2}<\ldots \\
& <\eta_{2 n-1}^{+}<\eta_{2 n-1}<\eta_{2 n-1}^{-}<\mu_{2 n-1}<\eta_{2 n}^{-}<\eta_{2 n}<\eta_{2 n}^{+}<\mu_{2 n}<\ldots .
\end{aligned}
$$

Due to Lemma 3, we obtain the following lemma: 
LEMMA 4. We have $D\left(\eta_{0}\right)=1, D\left(\eta_{2 n-1}^{+}\right) \geq-\frac{649}{576}, D\left(\eta_{2 n-1}\right) \geq \frac{5}{4}, D\left(\eta_{2 n-1}^{-}\right) \leq$ $-\frac{649}{576}, D\left(\mu_{2 n-1}\right) \geq 1, D\left(\eta_{2 n}^{-}\right) \leq-\frac{649}{576}, D\left(\eta_{2 n}\right) \geq \frac{5}{4}, D\left(\eta_{2 n}^{+}\right) \leq-\frac{649}{576}, D\left(\mu_{2 n}\right) \geq 1$ for $n \in \mathbf{N}$.

Using Lemma 2 and 4, we demonstrate the proof of Theorem 2 (II).

Proof of Theorem 2 (II). We fix a $c \in\left(-\frac{649}{576}, 1\right)$. For this $c$, we pick a large $n_{0} \in \mathbf{N}$ satisfying the statement of Lemma 2. We first shall show that the simple zero of $D(\lambda)-c$ inside $C_{7}(n)$ is real for $n \geq n_{0}$. By virtue of (22), we notice that the zeroes of $\Delta(\lambda)-\gamma$ and $\cos \sqrt{\lambda}-\gamma$ are close for a fixed $\gamma \in[-1,1]$ if $\lambda$ is enough large. Thus, for a fixed small $\varepsilon>0$, there exists some $n_{1}=n_{1}(\varepsilon) \in \mathbf{N}$ such that $\eta_{2 n} \in\left(2 n \pi+\frac{3}{2} \pi-\varepsilon, 2 n \pi+\frac{3}{2} \pi+\varepsilon\right)$, $\eta_{2 n}^{+} \in\left(2 n \pi+\gamma_{9}-\varepsilon, 2 n \pi+\gamma_{9}+\varepsilon\right), \mu_{2 n} \in(2(n+1) \pi-\varepsilon, 2(n+1) \pi+\varepsilon), \eta_{2 n+1}^{+} \in(2(n+$ 1) $\left.\pi+\gamma_{1}-\varepsilon, 2(n+1) \pi+\gamma_{1}+\varepsilon\right)$ and $\eta_{2 n+1} \in\left(2(n+1) \pi+\frac{\pi}{2}-\varepsilon, 2(n+1) \pi+\frac{\pi}{2}+\varepsilon\right)$ if $n \geq n_{1}$. Let the above number $\varepsilon>0$ be taken such that $\varepsilon<\min \left\{\left(\gamma_{j+1}-\gamma_{j}\right) / 2 \mid j=0,1,2, \ldots, 9\right\}$ and $n \geq \max \left\{n_{1}, n_{0}\right\}$. Since $D\left(\eta_{2 n}\right) \geq 1$ and $D\left(\eta_{2 n}^{+}\right) \leq-\frac{649}{576}$, we see by the intermediate value theorem that there exists at least one zero of $D(\lambda)-c$ in the interval $\left(\eta_{2 n}, \eta_{2 n}^{+}\right)$. Due to Lemma 2 , there are no zeroes of $D(\lambda)-c$ in $\left(2 n \pi+\gamma_{7}, 2 n \pi+\gamma_{8}\right)$. Hence, there exists at least one zero of $D(\lambda)-c$ in $\left(2 n \pi+\gamma_{8}, \eta_{2 n}^{+}\right)$.

We prove that $D\left(2 n \pi+\gamma_{9}\right) \leq-\frac{649}{576}$ by a contradiction. Seeking a contradiction, we assume that $D\left(2 n \pi+\gamma_{9}\right)>-\frac{649}{576}$. This implies that there exists some $c_{0} \in\left(-\frac{649}{576}, 1\right)$ such that the simple zero of $D(\lambda)-c$ is real for $c \in\left(c_{0}, 1\right)$ and the simple zero of $D(\lambda)-c$ is not real for $c \in\left(-\frac{649}{576}, c_{0}\right)$. Since $\eta_{2 n}^{+} \in\left(2 n \pi+\gamma_{9}-\varepsilon, 2 n \pi+\gamma_{9}+\varepsilon\right), D\left(\eta_{2 n}^{+}\right) \leq-\frac{649}{576}$ and our assumption $D\left(2 n \pi+\gamma_{9}\right)>-\frac{649}{576}$, we see by the intermediate value theorem that there exists at least one zero of $D(\lambda)-c$ in $\left(2 n \pi+\gamma_{9}, 2 n \pi+\gamma_{9}+\varepsilon\right)$ for any $c \in\left(-\frac{649}{576}, c_{0}\right)$. By Lemma 2 , we see that there is exactly one zero of $D(\lambda)-c$ in $C_{8}(n)$ for a fixed $c \in\left(-\frac{649}{576}, 1\right)$. This means that $D(\lambda) \leq-\frac{649}{576}$ in $\left(2 n \pi+\gamma_{9}+\varepsilon, 2(n+1), \pi\right)$ (Otherwise, there are at least two zeroes of $D(\lambda)-c$ in $C_{8}(n)$ for $c \in\left(-\frac{649}{576}, 1\right)$. This violates the statement of Lemma 2.). Next, we recall that $\mu_{2 n} \in(2(n+1) \pi-\varepsilon, 2(n+1) \pi+\varepsilon)$. This combined with the intermediate value theorem means that there exist at least one real zero of $D(\lambda)-c$ in $(2(n+1) \pi, 2(n+1) \pi+\varepsilon)$ for $c \in\left(-\frac{649}{576}, 1\right)$. By virtue of Lemma 2, we see that there exists exactly one zero of $D(\lambda)-c$ in $C_{1}(n+1)$ for $c \in\left(-\frac{649}{576}, 1\right)$. Thus, we have $D(\lambda) \geq 1$ in $\left(2(n+1) \pi+\varepsilon, 2(n+1) \pi+\gamma_{1}\right)$. We next take account of $\eta_{2 n+1}^{+} \in\left(2(n+1) \pi+\gamma_{1}-\varepsilon, 2(n+1) \pi+\gamma_{1}+\varepsilon\right)$ and $D\left(\eta_{2 n+1}^{+}\right) \leq$ $-\frac{649}{576}$. It follows by the intermediate value theorem that $D(\lambda)-c$ has at least one real zero in $\left(2(n+1) \pi+\gamma_{1}, 2(n+1) \pi+\gamma_{1}+\varepsilon\right)$ for $c \in\left(-\frac{649}{576}, 1\right)$. The number of zero of $D(\lambda)-c$ inside $C_{2}(n+1)$ for $c \in\left(-\frac{649}{576}, 1\right)$ is exactly one, due to Lemma 2 . So, we notice that $D(\lambda)-c$ has no zeroes in $\left(2(n+1) \pi+\gamma_{1}+\varepsilon, 2(n+1) \pi+\gamma_{2}\right)$ for $c \in\left(-\frac{649}{576}, 1\right)$. Moreover, there are no zeroes of $D(\lambda)-c$ in $\left(2(n+1) \pi+\gamma_{2}, 2(n+1) \pi+\gamma_{3}\right)$ for $c \in\left(-\frac{649}{576}, 1\right)$ because of Lemma 2. 
However, we notice that $D\left(\eta_{2 n+1}\right) \geq 1$ for $\eta_{2 n+1} \in\left(2(n+1) \pi+\frac{\pi}{2}-\varepsilon, 2(n+1) \pi+\frac{\pi}{2}+\varepsilon\right) \subset$ $\left(2(n+1) \pi+\gamma_{2}, 2(n+1) \pi+\gamma_{3}\right)$. This contradict the continuity of $D(\lambda)$ and hence the intermediate value theorem. This is why we see that $D\left(2 n \pi+\gamma_{9}\right) \leq-\frac{649}{576}$ and the simple zero of $D(\lambda)-c$ in $C_{7}(n)$ is real for $c \in\left(-\frac{649}{576}, 1\right)$. In a similar way, we see that the simple zero of $D(\lambda)-c$ in $C_{j}(n)$ is real for $c \in\left(-\frac{649}{576}, 1\right), n \geq n_{2}:=\max \left\{n_{1}, n_{0}\right\}$ and every $j=1,2,3, \ldots, 8$.

Furthermore, it follows by Lemma 2 and 4 that $D(\lambda)-c$ has exactly $8 n_{2}$ real zeroes in $C_{0}\left(n_{2}\right)$ for $c \in\left(-\frac{649}{576}, 1\right)$. Therefore, we conclude that the zeroes of $D(\lambda)-c$ are simple and real for $c \in\left(-\frac{649}{576}, 1\right)$.

To prove Theorem 2 (III), we make use of Laguerre's theorem. We quote it from [21].

DEFINITION 1. An entire function $f(z)$ is said to be of finite order if there is a positive number $A$ such that

$$
f(z)=\mathcal{O}\left(e^{r^{A}}\right) \text { as } \quad|z|=r \rightarrow \infty .
$$

The lower bound $\rho$ of numbers $A$ for which this is true is called the order of the function $f(z)$.

THEOREM 4 (Laguerre, see Section 8.52 in [21]). If $f(z)$ is an entire function, is real for a real $z$, of order less than 2 , with real zeroes, then the zeroes of $f^{\prime}(z)$ are also all real and are separated from each other by the zeroes of $f(z)$.

Proof of Theorem 2 (III), (IV), (V). We shall show the statement (III). It follows by (23) that the order of $D(\lambda)$ is less than 2 . Furthermore, $D(\lambda)$ has only simple real zero, due to Theorem 2 (II). Since $\theta(x, \lambda), \theta^{\prime}(x, \lambda), \varphi(x, \lambda), \varphi^{\prime}(x, \lambda)$ are entire in $\lambda \in \mathbf{C}$ and real for real $\lambda$, so is the function $D(\lambda)$. Utilizing Laguerre's theorem, we see that the zeroes of $D^{\prime}(\lambda)$ are also all real and are separated from each other by the zeroes of $D(\lambda)$. Taking account of Theorem 2 (I), we obtain the inequality (2). Since the zeroes of $D(\lambda)-c$ is simple for $c \in\left(-\frac{649}{576}, 1\right)$, we see that $D\left(\lambda_{3,2 n}\right) \geq 1$ and $D\left(\lambda_{3,2 n-1}\right) \leq-\frac{649}{576}<-1$ for any $n \in \mathbf{N}$.

Combining Lemma 2, 4 and Theorem (II), (III), we obtain (IV) and (V).

\section{Proof of Theorem 1.3}

We first introduce the monodromy matrix for $H$.

Definition 2. For $\lambda \in \mathbf{C} \backslash \sigma_{D}\left(H_{0}\right)$, let $\Theta(x, \lambda)=\left\{\Theta_{\alpha}(x, \lambda)\right\}_{\alpha \in \mathcal{Z}}$ and $\Phi(x, \lambda)=$ $\left\{\Phi_{\alpha}(x, \lambda)\right\}_{\alpha \in \mathcal{Z}}$ be the solutions to the equations

$$
\begin{aligned}
& -f_{\alpha}^{\prime \prime}(x)+q(x) f_{\alpha}(x)=\lambda f_{\alpha}(x), \quad x \in \Gamma_{\alpha} \simeq(0,1), \quad \alpha \in \mathcal{Z}, \\
& f_{n, 1}(1)=f_{n, 2}(1)=f_{n, 3}(0), \\
& -f_{n, 1}^{\prime}(1)+f_{n, 2}^{\prime}(1)+f_{n, 3}^{\prime}(0)=0, \\
& f_{n, 2}(1)=f_{n, 3}(1)=f_{n, 4}(0)=f_{n, 5}(0),
\end{aligned}
$$




$$
\begin{aligned}
& -f_{n, 2}^{\prime}(1)-f_{n, 3}^{\prime}(1)+f_{n, 4}^{\prime}(0)+f_{n, 5}^{\prime}(0)=0, \\
& f_{n, 4}(1)=f_{n, 5}(1)=f_{n, 6}(0)=f_{n, 7}(0), \\
& -f_{n, 4}^{\prime}(1)-f_{n, 5}^{\prime}(1)+f_{n, 6}^{\prime}(0)+f_{n, 7}^{\prime}(0)=0 . \\
& f_{n, 6}(1)=f_{n, 7}(1)=f_{n+1,1}(0), \\
& -f_{n, 6}^{\prime}(1)-f_{n, 7}^{\prime}(1)+f_{n+1,1}^{\prime}(0)=0
\end{aligned}
$$

for $n \in \mathbf{N}$ subject to the initial conditions

$$
\Theta_{0,1}(0, \lambda)=1, \quad \Theta_{0,1}^{\prime}(0, \lambda)=0, \quad \text { and } \quad \Phi_{0,1}(0, \lambda)=0, \quad \Phi_{0,1}^{\prime}(0, \lambda)=1,
$$

respectively. Then, we define the monodromy matrix with respect to $H$ as

$$
\mathcal{M}(\lambda)=\left(\begin{array}{ll}
\Theta_{1,1}(0, \lambda) & \Phi_{1,1}(0, \lambda) \\
\Theta_{1,1}^{\prime}(0, \lambda) & \Phi_{1,1}^{\prime}(0, \lambda)
\end{array}\right) .
$$

We abbreviate $\theta(1, \lambda), \theta^{\prime}(1, \lambda), \varphi(1, \lambda), \varphi^{\prime}(1, \lambda)$ and $\Delta(\lambda)$ to $\theta_{1}, \theta_{1}^{\prime}, \varphi_{1}, \varphi_{1}^{\prime}$ and $\Delta$, respectively. Then, the components of $\mathcal{M}(\lambda)$ are written by $\theta_{1}, \theta_{1}^{\prime}, \varphi_{1}, \varphi_{1}^{\prime}$ and $\Delta$ :

LEMMA 5. We have

$$
\begin{aligned}
& \Theta_{1,1}(0)=2 \Delta^{2}\left(2 \theta_{1}+\varphi_{1}^{\prime}\right) \theta_{1}-2 \Delta^{2}-2 \Delta \theta_{1}-\frac{\theta_{1}\left(2 \theta_{1}+\varphi_{1}^{\prime}\right)}{2}+\frac{1}{2}, \\
& \Theta_{1,1}^{\prime}(0)=\frac{2 \varphi_{1}^{\prime}}{\varphi_{1}} \Theta_{1,1}(0)-\frac{2}{\varphi_{1}}\left\{\left(2 \theta_{1}+\varphi_{1}^{\prime}\right) \Delta \theta_{1}-\Delta-\theta_{1}\right\} \\
& \Phi_{1,1}(0)=\Delta\left\{2 \Delta\left(2 \theta_{1}+\varphi_{1}^{\prime}\right) \varphi_{1}-2 \varphi_{1}\right\}-\frac{2 \theta_{1}+\varphi_{1}^{\prime}}{2} \varphi_{1}, \\
& \Phi_{1,1}^{\prime}(0)=\frac{2 \varphi_{1}^{\prime}}{\varphi_{1}} \Phi_{1,1}(0)-2 \Delta\left(2 \theta_{1}+\varphi_{1}^{\prime}\right)+2,
\end{aligned}
$$

$\operatorname{det} \mathcal{M}(\lambda)=1$ and $\operatorname{tr} \mathcal{M}(\lambda)=2 D(\lambda)$.

Proof. For $\lambda \in \mathbf{C} \backslash \sigma_{D}\left(H_{0}\right)$, any solution to $-f^{\prime \prime}(x)+q(x) f(x)=\lambda f(x)$ is given as

$$
f(x, \lambda)=f(0, \lambda) \theta(x, \lambda)+\frac{f(1, \lambda)-\theta(1, \lambda) f(0, \lambda)}{\varphi(1, \lambda)} \varphi(x, \lambda) .
$$

Thus, we have

$$
f_{\alpha}^{\prime}(0, \lambda)=\frac{f_{\alpha}(1, \lambda)-\theta(1, \lambda) f_{\alpha}(0, \lambda)}{\varphi(1, \lambda)} \quad \text { and } \quad f_{\alpha}^{\prime}(1, \lambda)=\frac{\varphi^{\prime}(1, \lambda) f_{\alpha}(1, \lambda)-f_{\alpha}(0, \lambda)}{\varphi(1, \lambda)}
$$

for a solution $f_{\alpha}(x, \lambda)$ to (31), where $\alpha \in \mathcal{Z}$. Substituting these for (33), we derive

$$
-\frac{\varphi_{1}^{\prime} f_{0,1}(1, \lambda)-f_{0,1}(0, \lambda)}{\varphi_{1}}+\frac{f_{0,2}(1, \lambda)-\theta_{1} f_{0,2}(0, \lambda)}{\varphi_{1}}+\frac{f_{0,3}(1, \lambda)-\theta_{1} f_{0,3}(0, \lambda)}{\varphi_{1}}=0 .
$$


We abbreviate $f_{\alpha}(1, \lambda)$ and $f_{\alpha}(0, \lambda)$ to $f_{\alpha}(1)$ and $f_{\alpha}(0)$, respectively. Then, we obtain

$$
-\left(2 \theta_{1}+\varphi_{1}^{\prime}\right) f_{0,1}(1)+f_{0,1}(0)+f_{0,2}(1)+f_{0,3}(1)=0
$$

because of (32). Similarly, it follows by using (34), (36) and (38) after substituting (44) for (35), (37) and (39) that

$$
\begin{aligned}
& -4 \Delta f_{0,2}(1)+f_{0,2}(0)+f_{0,3}(0)+f_{0,4}(1)+f_{0,5}(1)=0, \\
& -4 \Delta f_{0,4}(1)+f_{0,4}(0)+f_{0,5}(0)+f_{0,6}(1)+f_{0,7}(1)=0, \\
& -2 \varphi_{1}^{\prime} f_{0,6}(1)+f_{0,6}(0)+f_{0,7}(0)+\varphi_{1} f_{1,1}^{\prime}(0)=0 .
\end{aligned}
$$

Notice that $\Theta_{0,1}(x, \lambda)=\theta(x, \lambda)$. Thus, (45) implies that

$$
-\left(2 \theta_{1}+\varphi_{1}^{\prime}\right) \theta_{1}+1+2 \Theta_{0,2}(1)=0
$$

because of $\Theta_{0,2}(1)=\Theta_{0,3}(1)$. Since $\theta_{0,2}(0)=\Theta_{0,3}(0)=\theta_{1}$ and $\Theta_{0,4}(1)=\Theta_{0,5}(1)$, it follows by (46) that

$$
-4 \Delta \Theta_{0,2}(1)+2 \theta_{1}+2 \Theta_{0,4}(1)=0 .
$$

Since $\Theta_{0,4}(0)=\Theta_{0,5}(0)$ and $\Theta_{0,6}(1)=\Theta_{0,7}(1)=\Theta_{1,1}(0)$, we have

$$
-4 \Delta \Theta_{0,4}(1)+2 \Theta_{0,2}(1)+2 \Theta_{1,1}(0)=0
$$

by (47). We derive (40) by (49), (50) and (51). Furthermore, we have

$$
\Theta_{1,1}^{\prime}(0)=\frac{2 \varphi_{1}^{\prime}}{\varphi_{1}} \Theta_{1,1}(0)-\frac{2}{\varphi_{1}} \Theta_{0,4}(1)
$$

by virtue of $\Theta_{0,6}(0)=\Theta_{0,7}(0)=\Theta_{0,4}(1)$ and $\Theta_{0,6}(1)=\Theta_{1,1}(0)$. This combined with (49), (50) produces (41). In a similar way, we also derive (42) and (43). Direct calculations yield both $\operatorname{det} \mathcal{M}(\lambda)=1$ and $\operatorname{tr} \mathcal{M}(\lambda)=2 D(\lambda)$.

Proof of Theorem 1.3 (I), (II) AND (III). It follows by $\operatorname{det} \mathcal{M}(\lambda)=1$ and $\operatorname{tr} \mathcal{M}(\lambda)=2 D(\lambda)$ that $\operatorname{det}(\mathcal{M}(\lambda) \pm I)=2(1 \pm D(\lambda))$, where $I$ stands for the unit matrix. So, we have $\sigma\left(H_{a p}\right)=\{\lambda \in \mathbf{R} \mid \quad D(\lambda)=-1\}$ and $\sigma\left(H_{p}\right)=\{\lambda \in \mathbf{R} \mid \quad D(\lambda)=1\}$. Thus, we see that $z_{0}^{+}=\lambda_{3,0}^{+}, z_{n}^{ \pm}=\lambda_{3,2 n}^{ \pm}$and $x_{n}^{ \pm}=\lambda_{3,2 n-1}^{ \pm}$for $n \in \mathbf{N}$. Hence, we have (I) by Theorem 2 (V). This statement and Theorem 1 yield (II) and (III).

To make sure of Theorem 3 (IV), we use the following lemma:

LeMma 6. (I) The sequence $\left\{z_{n}^{ \pm}\right\}$satisfies the asymptotics

$$
\begin{aligned}
& \sqrt{z_{4 n}^{ \pm}}=u_{4 n}^{ \pm}+\frac{q_{0}}{2 u_{4 n}^{ \pm}}+o\left(\frac{1}{n}\right), \\
& \sqrt{z_{4 n-3}^{ \pm}}=u_{4 n-3}^{ \pm}+\frac{q_{0}}{2 u_{4 n-3}^{ \pm}}+o\left(\frac{1}{n^{2}}\right),
\end{aligned}
$$




$$
\begin{aligned}
& \sqrt{z_{4 n-2}^{ \pm}}=u_{4 n-2}^{ \pm}+\frac{q_{0}}{2 u_{4 n-2}^{ \pm}}+o\left(\frac{1}{n}\right), \\
& \sqrt{z_{4 n-1}^{ \pm}}=u_{4 n-1}^{ \pm}+\frac{q_{0}}{2 u_{4 n-1}^{ \pm}}+o\left(\frac{1}{n^{2}}\right) \text { as } n \rightarrow \infty .
\end{aligned}
$$

(II) The sequence $\left\{x_{n}^{ \pm}\right\}$satisfies the asymptotics

$$
\sqrt{x_{n}^{ \pm}}=v_{n}^{ \pm}+\frac{q_{0}}{2 v_{n}^{ \pm}}+o\left(\frac{1}{n^{2}}\right) \quad \text { as } \quad n \rightarrow \infty .
$$

Proof. Recall (30). Noting that $\Delta^{2}(\lambda)-\Delta_{-}^{2}(\lambda)=\theta(1, \lambda) \varphi^{\prime}(1, \lambda)$, we have

$$
D(\lambda)=9 \Delta^{4}(\lambda)-\left(\frac{37}{4}+\frac{3}{4} \Delta_{-}^{2}(\lambda)\right) \Delta^{2}(\lambda)+\frac{5}{4} .
$$

Solving the quartic equation $D(\lambda)-1=0$ with respect to $\Delta(\lambda)$, we obtain $\Delta(\lambda)=$ $\Delta_{1}(\lambda), \Delta_{2}(\lambda), \Delta_{3}(\lambda), \Delta_{4}(\lambda)$, where

$$
\begin{aligned}
& \Delta_{1}(\lambda)=\sqrt{\frac{37+3 \Delta_{-}^{2}(\lambda)+\sqrt{\left(37+3 \Delta_{-}^{2}(\lambda)\right)^{2}-144}}{72}}, \\
& \Delta_{2}(\lambda)=\sqrt{\frac{37+3 \Delta_{-}^{2}(\lambda)-\sqrt{\left(37+3 \Delta_{-}^{2}(\lambda)\right)^{2}-144}}{72}}, \\
& \Delta_{3}(\lambda)=-\sqrt{\frac{37+3 \Delta_{-}^{2}(\lambda)-\sqrt{\left(37+3 \Delta_{-}^{2}(\lambda)\right)^{2}-144}}{72}}, \\
& \Delta_{4}(\lambda)=-\sqrt{\frac{37+3 \Delta_{-}^{2}(\lambda)+\sqrt{\left(37+3 \Delta_{-}^{2}(\lambda)\right)^{2}-144}}{72}} .
\end{aligned}
$$

Note that $\Delta_{1}(\lambda) \rightarrow 1, \Delta_{2}(\lambda) \rightarrow \frac{1}{6}, \Delta_{3}(\lambda) \rightarrow \frac{1}{6}, \Delta_{4}(\lambda) \rightarrow-1$ as $\lambda \rightarrow \infty$ because of $\Delta_{-}(\lambda) \rightarrow 0$ as $\lambda \rightarrow \infty$. Reset $\Omega(a, r)=\{\lambda \in \mathbf{C}|| \sqrt{\lambda}-a \mid<r\}$ for $a \in \mathbf{C}$ and $r>0$. It follows by Lemma 1 and Theorem 2 (IV) that $z_{4 n}^{ \pm} \in \Omega\left(2 n \pi, \frac{\pi}{8}\right), z_{4 n-1}^{ \pm} \in \Omega\left(2 n \pi-\frac{\pi}{2}, \frac{\pi}{8}\right)$, $z_{4 n-2}^{ \pm} \in \Omega\left(2 n \pi-\pi, \frac{\pi}{8}\right)$ and $z_{4 n-3}^{ \pm} \in \Omega\left(2 n \pi-\frac{3}{2} \pi, \frac{\pi}{8}\right)$ if $n \in \mathbf{N}$ is enough large. On the other hand, we see that $\left(u_{4 n-3}^{ \pm}\right)^{2} \in \Omega\left(2 n \pi-\frac{3}{2}, \frac{\pi}{8}\right),\left(u_{4 n-2}^{ \pm}\right)^{2} \in \Omega\left(2 n \pi-\pi, \frac{\pi}{8}\right),\left(u_{4 n-1}^{ \pm}\right)^{2} \in$ $\Omega\left(2 n \pi-\frac{\pi}{2}, \frac{\pi}{8}\right)$ and $\left(u_{4 n}^{ \pm}\right)^{2} \in \Omega\left(2 n \pi, \frac{\pi}{8}\right)$ for any $n \in \mathbf{N}$. Thus, we see that $\left|\sqrt{z_{4 n}^{ \pm}}-u_{4 n}^{ \pm}\right|<\frac{\pi}{4}$, $\left|\sqrt{z_{4 n-1}^{ \pm}}-u_{4 n-1}^{ \pm}\right|<\frac{\pi}{4},\left|\sqrt{z_{4 n-2}^{ \pm}}-u_{4 n-2}^{ \pm}\right|<\frac{\pi}{4}$ and $\left|\sqrt{z_{4 n-3}^{ \pm}}-u_{4 n-3}^{ \pm}\right|<\frac{\pi}{4}$ for large $n \in \mathbf{N}$. 
According to [10], we see that

$$
\Delta(\lambda)=\cos \chi(\lambda), \quad \chi(\lambda)=\sqrt{\lambda}-\frac{q_{0}}{2 \sqrt{\lambda}}+\frac{o(1)}{\lambda} \quad \text { as } \quad|\lambda| \rightarrow \infty .
$$

Thus, we see that $\chi\left(z_{n}^{ \pm}\right)=a_{n}^{ \pm}+i b_{n}^{ \pm}$, where

$$
a_{n}^{ \pm}=\sqrt{z_{n}^{ \pm}}-\frac{q_{0}}{2 \sqrt{z_{n}^{ \pm}}}+\frac{o(1)}{z_{n}^{ \pm}} \in \mathbf{R} \quad \text { and } \quad b_{n}^{ \pm}=\frac{o(1)}{z_{n}^{ \pm}} \in \mathbf{R} \quad \text { as } \quad n \rightarrow \infty .
$$

We see that $\Delta_{2}\left(z_{4 n-3}^{-}\right) \rightarrow \frac{1}{6}$ and $\Delta_{2}\left(z_{4 n-1}^{+}\right) \rightarrow \frac{1}{6}$ as $n \rightarrow \infty$ as well as

$$
\begin{gathered}
\Delta_{2}\left(z_{4 n-3}^{-}\right)=\cos \chi\left(z_{4 n-3}^{-}\right)=\cos a_{4 n-3}^{-} \cosh b_{4 n-3}^{-}-i \sin a_{4 n-3}^{-} \sinh b_{4 n-3}^{-}, \\
\Delta_{2}\left(z_{4 n-1}^{+}\right)=\cos \chi\left(z_{4 n-1}^{+}\right)=\cos a_{4 n-1}^{+} \cosh b_{4 n-1}^{+}-i \sin a_{4 n-1}^{+} \sinh b_{4 n-1}^{+} .
\end{gathered}
$$

These imply that $\cos a_{4 n-3}^{-} \rightarrow \frac{1}{6}$ and $\cos a_{4 n-1}^{+} \rightarrow \frac{1}{6}$ as $n \rightarrow \infty$. This combined with $\left|\sqrt{z_{4 n-3}^{-}}-u_{4 n-3}^{-}\right|<\frac{\pi}{4}$ and $\left|\sqrt{z_{4 n-1}^{+}}-u_{4 n-1}^{+}\right|<\frac{\pi}{4}$ means that

$$
\begin{gathered}
\sqrt{z_{4 n-3}^{-}}-u_{4 n-3}^{-}-\frac{q_{0}}{2 \sqrt{z_{4 n-3}^{-}}}+\frac{o(1)}{z_{4 n-3}^{-}} \rightarrow 0, \\
\sqrt{z_{4 n-1}^{+}}-u_{4 n-1}^{+}-\frac{q_{0}}{2 \sqrt{z_{4 n-1}^{+}}}+\frac{o(1)}{z_{4 n-1}^{+}} \rightarrow 0
\end{gathered}
$$

as $n \rightarrow \infty$. Similarly (and collectively), we have

$$
\sqrt{z_{n}^{ \pm}}-u_{n}^{ \pm}-\frac{q_{0}}{2 \sqrt{z_{n}^{ \pm}}}+\frac{o(1)}{z_{n}^{ \pm}} \rightarrow 0 \quad \text { as } \quad n \rightarrow \infty .
$$

Thus, we have

$$
\varepsilon_{n}^{ \pm}=\sqrt{z_{n}^{ \pm}}-u_{n}^{ \pm}-\frac{q_{0}}{2 u_{n}^{ \pm}}+\frac{o(1)}{n^{2}} \rightarrow 0 \quad \text { as } \quad n \rightarrow \infty .
$$

Owing to Taylor's theorem, there exists some $\theta_{n}^{ \pm} \in(0,1)$ such that

$$
\begin{aligned}
\cos \chi\left(z_{n}^{ \pm}\right) & =\cos \left(u_{n}^{ \pm}+\varepsilon_{n}^{ \pm}\right) \\
& =\cos u_{n}^{ \pm}-\left(\sin u_{n}^{ \pm}\right) \varepsilon_{n}^{ \pm}-\frac{\cos u_{n}^{ \pm}}{2}\left(\varepsilon_{n}^{ \pm}\right)^{2}+\frac{\sin \left(u_{n}^{ \pm}+\varepsilon_{n}^{ \pm} \theta_{n}^{ \pm}\right)}{6}\left(\varepsilon_{n}^{ \pm}\right)^{3}
\end{aligned}
$$

and hence

$$
\begin{aligned}
\cos \chi\left(z_{4 n}^{ \pm}\right) & =1-\frac{1}{2}\left(\varepsilon_{4 n}^{ \pm}\right)^{2}\left(1+\mathcal{O}\left(\varepsilon_{4 n}^{ \pm}\right)\right), \\
\cos \chi\left(z_{4 n-2}^{ \pm}\right) & =-1+\frac{1}{2}\left(\varepsilon_{4 n-2}^{ \pm}\right)^{2}\left(1+\mathcal{O}\left(\varepsilon_{4 n-2}^{ \pm}\right)\right),
\end{aligned}
$$




$$
\begin{aligned}
& \left.\cos \chi\left(z_{4 n-1}^{ \pm}\right)\right)= \pm \frac{1}{6}+\left(\sin \left(\arccos \left( \pm \frac{1}{6}\right)\right)\right) \varepsilon_{4 n-1}^{ \pm}\left(1+\mathcal{O}\left(\varepsilon_{4 n-1}^{ \pm}\right)\right), \\
& \left.\cos \chi\left(z_{4 n-3}^{ \pm}\right)\right)=\mp \frac{1}{6}-\left(\sin \left(\arccos \left(\mp \frac{1}{6}\right)\right)\right) \varepsilon_{4 n-3}^{ \pm}\left(1+\mathcal{O}\left(\varepsilon_{4 n-3}^{ \pm}\right)\right) .
\end{aligned}
$$

We shall show that

$$
\cos \chi\left(z_{4 n}^{ \pm}\right)=1+o\left(\frac{1}{n^{2}}\right) \quad \text { as } n \rightarrow \infty .
$$

It follows by (20) and (21) that

$$
\Delta_{-}(\lambda)=-\frac{S(\lambda)}{\sqrt{\lambda}}+\frac{\mathcal{O}\left(e^{\mid \Im \sqrt{\lambda \mid}}\right)}{|\lambda|} \quad \text { as } \quad \lambda \rightarrow \infty .
$$

Thus, we have $\Delta_{-}^{2}\left(z_{4 n}^{ \pm}\right)=o\left(\frac{1}{n^{2}}\right)$ as $n \rightarrow \infty$. We now utilize Taylor's theorem for

$$
\cos \chi\left(z_{4 n}^{ \pm}\right)=\Delta_{1}\left(z_{4 n}^{ \pm}\right)=\sqrt{\frac{37+3 \Delta_{-}^{2}\left(z_{4 n}^{ \pm}\right)+\sqrt{\left(37+3 \Delta_{-}^{2}\left(z_{4 n}^{ \pm}\right)\right)^{2}-144}}{72}} .
$$

Thus, for any $x \in \mathbf{R}$, there exists some $\theta \in(0,1)$ such that

$$
\sqrt{(37+x)^{2}-144}=35+\frac{\theta x+37}{\sqrt{(37+\theta x)^{2}-144}} x .
$$

Hence, we have

$$
\sqrt{\left(37+3 \Delta_{-}^{2}\left(z_{4 n}^{ \pm}\right)\right)^{2}-144}=35+o\left(\frac{1}{n^{2}}\right) \quad \text { as } \quad n \rightarrow \infty
$$

and hence

$$
\cos \chi\left(z_{4 n}^{ \pm}\right)=\sqrt{\frac{37+o\left(\frac{1}{n^{2}}\right)+35+o\left(\frac{1}{n^{2}}\right)}{72}}=\sqrt{1+o\left(\frac{1}{n^{2}}\right)}=1+o\left(\frac{1}{n^{2}}\right) \quad \text { as } n \rightarrow \infty .
$$

This combined with (59) means that $\varepsilon_{4 n}^{ \pm}=o\left(\frac{1}{n}\right)$ as $n \rightarrow \infty$. Likewise, we obtain $\varepsilon_{4 n-2}^{ \pm}=$ $o\left(\frac{1}{n}\right), \varepsilon_{4 n-1}^{ \pm}=o\left(\frac{1}{n^{2}}\right)$ and $\varepsilon_{4 n-3}^{ \pm}=o\left(\frac{1}{n^{2}}\right)$ as $n \rightarrow \infty$. By the definition of $\varepsilon_{n}^{ \pm}$, we derive (53), (54), (55) and (56). The proof of the statement (II) is much alike.

Proof OF THEOREM 3 (IV). Since $\lambda_{3,0}^{+}=z_{0}^{+}, \lambda_{3,2 n-1}^{ \pm}=x_{n}^{ \pm}$and $\lambda_{3,2 n}^{ \pm}=z_{n}^{ \pm}$for any $n \in \mathbf{N}$, Lemma 6 directly means (3), (4), (5), (7), (8) and (9) as well as $\lambda_{3,8 n}^{ \pm}=\left(u_{4 n}^{ \pm}\right)^{2}+q_{0}+$ $o(1)$ and $\lambda_{3,8 n-4}^{ \pm}=\left(u_{4 n-2}^{ \pm}\right)^{2}+q_{0}+o(1)$ as $n \rightarrow \infty$. Below, we investigate the part of $o(1)$ of these.

Since $\sqrt{\lambda_{3,8 n}^{ \pm}}=u_{4 n}^{ \pm}+\frac{q_{0}}{2 u_{4 n}^{ \pm}}+o\left(\frac{1}{n}\right)$ as $n \rightarrow \infty$, we consider $\lambda$ satisfying $\sqrt{\lambda}=$ $2 n \pi+\mathcal{O}\left(\frac{1}{n}\right)$ as $n \rightarrow \infty$. We define $q_{c, j, n}=\int_{0}^{1}(1-2 t)^{j} q(t) \cos 2 n \pi t d t$ and 
$q_{s, j, n}=\int_{0}^{1}(1-2 t)^{j} q(t) \sin 2 n \pi t d t$ for $j \in \mathbf{N} \cup\{0\}$. Let $\dot{f}(\lambda)$ imply that the derivative of $f$ with respect to $\lambda$ for a differentiable function $f(\lambda)$. Using the definition of $S(\lambda)$ and

$$
\dot{S}(\lambda)=\frac{1}{2 \sqrt{\lambda}} \int_{0}^{1}(1-2 t) q(t) \cos \sqrt{\lambda}(1-2 t) d t,
$$

we have

$$
S(\lambda)=-\left(q_{s, 0,2 n}+\mathcal{O}\left(\frac{1}{n}\right)\right), \quad \dot{S}(\lambda)=\frac{q_{c, 1,2 n}+\mathcal{O}\left(\frac{1}{n}\right)}{4 n \pi}
$$

if $\sqrt{\lambda}=2 n \pi+\mathcal{O}\left(\frac{1}{n}\right)$ as $n \rightarrow \infty$. These together with (63) yield

$$
\Delta_{-}(\lambda)=\frac{q_{s, 0,2 n}+\mathcal{O}\left(\frac{1}{n}\right)}{4 n \pi}, \dot{\Delta}_{-}(\lambda)=-\frac{q_{c, 1,2 n}+\mathcal{O}\left(\frac{1}{n}\right)}{(4 n \pi)^{2}}
$$

if $\sqrt{\lambda}=2 n \pi+\mathcal{O}\left(\frac{1}{n}\right)$ as $n \rightarrow \infty$. Furthermore, we derive

$$
\Delta(\lambda)=1+\mathcal{O}\left(\frac{1}{n^{2}}\right), \quad \dot{\Delta}(\lambda)=\mathcal{O}\left(\frac{1}{n^{2}}\right), \quad \ddot{\Delta}(\lambda)=-\frac{1+\mathcal{O}\left(\frac{1}{n}\right)}{(4 n \pi)^{2}} .
$$

Let $\left\{\lambda_{n}\right\}_{n=1}^{\infty}$ be the increasing sequence consists of zeroes of $\dot{\Delta}(\lambda)$. We have

$$
\dot{D}(\lambda)=36 \Delta^{3}(\lambda) \dot{\Delta}(\lambda)-\frac{3}{2} \Delta_{-}(\lambda) \dot{\Delta}_{-}(\lambda) \Delta^{2}(\lambda)-\frac{3}{2} \Delta_{-}^{2}(\lambda) \Delta(\lambda) \dot{\Delta}(\lambda)
$$

by virtue of (58). We put $\varepsilon_{3,8 n}^{ \pm}=\lambda_{3,8 n}^{ \pm}-\lambda_{2 n}$ and $\varepsilon_{2 n}^{ \pm}=\lambda_{2 n}^{ \pm}-\lambda_{2 n}$ for $n \in \mathbf{N}$. Then, we have $\varepsilon_{3,8 n}^{ \pm} \rightarrow 0$ and $\varepsilon_{2 n}^{ \pm} \rightarrow 0$ as $n \rightarrow \infty$ because $\lambda_{3,8 n}^{ \pm}=4 n^{2} \pi^{2}+q_{0}+o(1), \lambda_{2 n}=$ $4 n^{2} \pi^{2}+q_{0}+\ell^{2}(n)$ and $\lambda_{n}^{ \pm}=n^{2} \pi^{2}+q_{0} \pm\left|\hat{q}_{n}\right|+\mathcal{O}\left(\frac{1}{n}\right)$ as $n \rightarrow \infty$, which appear from (53) and $[6,8]$. Utilizing Taylor's theorem, we have

$$
\Delta\left(\lambda_{3,8 n}^{ \pm}\right)=\Delta\left(\lambda_{2 n}\right)+\dot{\Delta}\left(\lambda_{2 n}\right) \varepsilon_{3,8 n}^{ \pm}+\frac{\ddot{\Delta}\left(\lambda_{2 n}\right)}{2}\left(\varepsilon_{3,8 n}^{ \pm}\right)^{2}+\frac{\dddot{\Delta}\left(\lambda_{2 n}\right)}{6}\left(\varepsilon_{3,8 n}^{ \pm}\right)^{3}\left(1+\mathcal{O}\left(\varepsilon_{3,8 n}^{ \pm}\right)\right) .
$$

Thus, $\dot{\Delta}\left(\lambda_{2 n}\right)=0$ means that $\Delta\left(\lambda_{3,6 n}^{ \pm}\right)=\Delta\left(\lambda_{2 n}\right)+A_{3,2 n}^{ \pm}$, where

$$
A_{3,2 n}^{ \pm}=\frac{\ddot{\Delta}\left(\lambda_{2 n}\right)}{2}\left(\varepsilon_{3,8 n}^{ \pm}\right)^{2}\left(1+\mathcal{O}\left(\frac{\varepsilon_{3,8 n}^{ \pm}}{n^{2}}\right)\right)
$$

as $n \rightarrow \infty$. It follows by Taylor's theorem that $1=\Delta\left(\lambda_{2 n}^{ \pm}\right)=\Delta\left(\lambda_{2 n}\right)+A_{2 n}^{ \pm}$, where

$$
A_{2 n}^{ \pm}=\frac{\ddot{\Delta}\left(\lambda_{2 n}\right)}{2}\left(\varepsilon_{2 n}^{ \pm}\right)^{2}\left(1+\mathcal{O}\left(\frac{\varepsilon_{2 n}^{ \pm}}{n^{2}}\right)\right)
$$

as $n \rightarrow \infty$. Thus, we obtain $\Delta\left(\lambda_{3,8 n}^{ \pm}\right)=1+A_{3,2 n}^{ \pm}-A_{2 n}^{ \pm}$. Using $D\left(\lambda_{3,8 n}^{ \pm}\right)=1$ and (58), we 
have

$$
9 \Delta^{4}\left(\lambda_{3,8 n}^{ \pm}\right)-\left(\frac{37}{4}+\frac{3}{4} \Delta_{-}^{2}\left(\lambda_{3,8 n}^{ \pm}\right)\right) \Delta^{2}(\lambda)+\frac{1}{4}=0
$$

Substituting $\Delta\left(\lambda_{3,8 n}^{ \pm}\right)=1+A_{3,2 n}^{ \pm}-A_{2 n}^{ \pm}$and $\Delta_{-}\left(\lambda_{3,8 n}^{ \pm}\right)=-\frac{q_{s, 0,2 n}+\mathcal{O}\left(\frac{1}{n}\right)}{(4 n \pi)^{2}}$ into this, we obtain

$$
9\left(1+A_{3,2 n}^{ \pm}-A_{2 n}^{ \pm}\right)^{4}-\left(\frac{37}{4}+\frac{3}{4}\left(\frac{q_{s, 0,2 n}+\mathcal{O}\left(\frac{1}{n}\right)}{(4 n \pi)^{2}}\right)^{2}\right)\left(1+A_{3,2 n}^{ \pm}-A_{2 n}^{ \pm}\right)^{2}+\frac{1}{4}=0 .
$$

Using $\ddot{\Delta}(\lambda)=-\frac{1+\mathcal{O}\left(\frac{1}{n}\right)}{(4 n \pi)^{2}}$, we have

$$
A_{3,2 n}^{ \pm}=-\frac{1+\mathcal{O}\left(\frac{1}{n}\right)}{2(4 n \pi)^{2}}\left(\varepsilon_{3,8 n}^{ \pm}\right)^{2}\left(1+\mathcal{O}\left(\frac{\varepsilon_{3,8 n}^{ \pm}}{n^{2}}\right)\right) \text { and } A_{2 n}^{ \pm}=-\frac{1+\mathcal{O}\left(\frac{1}{n}\right)}{2(4 n \pi)^{2}}\left(\varepsilon_{2 n}^{ \pm}\right)^{2}\left(1+\mathcal{O}\left(\frac{\varepsilon_{2 n}^{ \pm}}{n^{2}}\right)\right)
$$

as $n \rightarrow \infty$. Putting $B_{2 n}^{ \pm}=\frac{\left(\varepsilon_{2 n}^{ \pm}\right)^{2}-\left(\varepsilon_{3,8 n}^{ \pm}\right)^{2}}{2(4 n \pi)^{2}}$, we have

$$
A_{3,2 n}^{ \pm}-A_{2 n}^{ \pm}=B_{2 n}^{ \pm}+o\left(\frac{1}{n^{3}}\right)
$$

as $n \rightarrow \infty$. Noting $B_{2 n}^{ \pm}=o\left(\frac{1}{n^{2}}\right)$ as $n \rightarrow \infty$ and substituting (67) for (66), we have

$$
B_{2 n}^{ \pm}-\frac{3}{70} \cdot\left(\frac{q_{s, 0,2 n}+\mathcal{O}\left(\frac{1}{n}\right)}{4 n \pi}\right)^{2}+o\left(\frac{1}{n^{3}}\right)=0
$$

as $n \rightarrow \infty$. It follows by the definition of $B_{2 n}^{ \pm}$that

$$
\left(\varepsilon_{2 n}^{ \pm}\right)^{2}-\left(\varepsilon_{3,8 n}^{ \pm}\right)^{2}-\frac{3}{35}\left(q_{s, 0,2 n}+\mathcal{O}\left(\frac{1}{n}\right)\right)^{2}+o\left(\frac{1}{n}\right)=0 \quad \text { as } \quad n \rightarrow \infty .
$$

Since $q_{s, 0,2 n}=\Im \hat{q}_{2 n}$, we see that $\left|q_{s, 0,2 n}\right|=\mathcal{O}\left(\hat{q}_{2 n}\right)$ as $n \rightarrow \infty$. We note that $\lambda_{n}^{ \pm}=$ $n^{2} \pi^{2}+q_{0} \pm\left|\hat{q}_{n}\right|+\mathcal{O}\left(\frac{1}{n}\right)$ and $\lambda_{n}=n^{2} \pi^{2}+q_{0}+\mathcal{O}\left(\frac{1}{n}\right)$, which follow from [15] and [8]. Thus, we see that $\varepsilon_{2 n}^{ \pm}= \pm\left|\hat{q}_{2 n}\right|+\mathcal{O}\left(\frac{1}{n}\right)$ as $n \rightarrow \infty$. This combined with (68) yields $\left(\varepsilon_{3,8 n}^{ \pm}\right)^{2}=$ $\left|\hat{q}_{2 n}\right|^{2}-\frac{3}{35} q_{2,0,2 n}^{2}+\mathcal{O}\left(\frac{1}{n}\right)$ and hence

$$
\varepsilon_{3,8 n}^{ \pm}= \pm \sqrt{\left|\hat{q}_{2 n}\right|^{2}-\frac{3}{35} q_{2,0,2 n}^{2}}+\mathcal{O}\left(\frac{1}{n}\right) \quad \text { as } \quad n \rightarrow \infty .
$$

This combined with $\lambda_{n}=n^{2} \pi^{2}+q_{0}+\mathcal{O}\left(\frac{1}{n}\right)$ as $n \rightarrow \infty$ yields (10). In a similar way, we also obtain (6). 
AcKnowledgment. This work is supported by Grant-in-Aid for Young Scientists (2580085), Japan Society for Promotion of Science. The author thanks the referee for taking time from his/her busy schedule to read a manuscript carefully. By the report form the referee, the author could improve this paper.

\section{References}

[1] G. Berkolaiko and P. KUChMEnt, Introduction to quantum graphs, AMS, Providence, RI, 2012.

[2] P. Duclos, P. EXner and O. Turek, On the spectrum of a bent chain graph, J. Phys, A: Math. Theor. 41 (2008), 41520618 pp.

[ 3 ] R. CARLSON, Hill's equation for a homogeneous tree, Electron. J. Differential Equations 23 (1997), 1-30.

[ 4 ] T. CheOn, P. EXNer and O. TUReK, Spectral filtering in quantum Y-junction, J. Phys. Soc. Jpn. 78 (2009), 124004.

[ 5 ] NGoc T. Do and P. Kuchment, Quantum graph spectra of a graphyne structure, Nanoscale Syst. 2 (2013), 107-123.

[6] J. Garnett and E. Trubowitz, Gaps and bands of one dimensional periodic Schrödinger operators, Comment. Math. Helv. 59 (1984), 258-312.

[ 7 ] C. GÉRARD and F. NiER, The Mourre theory for analytically fibered operators, J. Func. Anal. 152 (1998), no.1, 202-219.

[ 8 ] E. Korotyaev, The inverse problem for the Hill operator. I, Int. Math. Rs. Not. 3 (1997), 113-125.

[9] E. Korotyaev, Effective masses for zigzag nanotubes in magnetic fields, Lett. Math. Phys. 83 (2008), no.1, $83-95$.

[10] P. Kargaev and E. Korotyaev, Effective masses and conformal mappings, Comm. Math. Phys. 169 (1995), 597-625.

[11] E. Korotyaev and I. LobAnov, Schrödinger operators on zigzag nanotubes, Ann. Henri Poincaré 8 (2007), 1151-1176.

[12] V. Kostrykin and R. Schrader, Kirchhoff's rule for quantum wires, J. Phys. A: Math. Gen. 32 (1999), 595-630.

[13] P. Kuchment and O. Post, On the spectra of carbon nano-structures, Commun. Math. Phys. 275 (2007), 805-826.

[14] W. Magnus and S. WinkLer, Hill's Equation, Wiley, 1966.

[15] V. MARChenKo and I. OStrovski, A characterization of the spectrum of the Hill operator, Math. USSR Sb. 26 (1975), 493-554.

[16] H. NIIKUNI, Decisiveness of the spectral gaps of periodic Schrödinger operators on the dumbbell-like metric graph, Opuscula Mathematica 35-2(2015), 199-234.

[17] K. PAnkrashinin, Spectra of Schrödinger operators on equilateral quantum graphs, Lett. Math. Phys. 77 (2006), no. 2, 139-154.

[18] K. PANKRASHKIn, Localization effects in a periodic quantum graph with magnetic field and spin-orbit interaction, J. Math. Phys. 47 (2006), no.11, 112105, 17 pp.

[19] J. Poschel and E. Trubowitz, Inverse spectral theory, Academic Press, Orlando, 1987.

[20] M. ReED and B. Simon, Methods of modern mathematical physics, IV. Analysis of operators, Academic Press, New York, 1978.

[21] E. Titchmarsh, The theory of functions, Sec. ed., Oxford Univ. Press, London, 1975. 
Present Address:

Maebashi Institute of Technology,

460-1 KAMISAdori, MaEbashi City, GunMa 371-0816, JAPAN.

e-mail: niikuni@maebashi-it.ac.jp 